\title{
Locality Optimized Unstructured Mesh Algorithms on GPUs
}

\author{
A.A. Sulyok ${ }^{\mathrm{a}, \mathrm{b}, *}$, G.D. Balogh ${ }^{\mathrm{a}, \mathrm{b}}$, I.Z. Reguly ${ }^{\mathrm{a}}$, G.R. Mudalige ${ }^{\mathrm{c}}$ \\ ${ }^{a}$ Faculty of Information Technology and Bionics, Pázmány Péter Catholic University, \\ Budapest, Hungary \\ ${ }^{b}$ 3in-PPCU Research Group, Pázmány Péter Catholic University, Esztergom, Hungary \\ ${ }^{c}$ Department of Computer Science, University of Warwick, Coventry, United Kingdom
}

\begin{abstract}
Unstructured-mesh based numerical algorithms such as finite volume and finite element algorithms form an important class of applications for many scientific and engineering domains. The key difficulty in achieving higher performance from these applications is the indirect accesses that lead to data-races when parallelized. Current methods for handling such data-races lead to reduced parallelism and suboptimal performance. Particularly on modern many-core architectures, such as GPUs, that has increasing core/thread counts, reducing data movement and exploiting memory locality is vital for gaining good performance.

In this work we present novel locality-exploiting optimizations for the efficient execution of unstructured-mesh algorithms on GPUs. Building on a two-layered coloring strategy for handling data races, we introduce novel reordering and partitioning techniques to further improve efficient execution. The new optimizations are then applied to several well established unstructuredmesh applications, investigating their performance on NVIDIA's latest P100 and V100 GPUs. We demonstrate significant speedups (1.1-1.75×) compared to the state-of-the-art. A range of performance metrics are benchmarked including runtime, memory transactions, achieved bandwidth performance, GPU
\end{abstract}

\footnotetext{
* Corresponding author

Email address: sulyok.andras.attila@itk.ppke.hu (A.A. Sulyok)
}

Preprint submitted to Journal of Parallel and Distributed Computing

September 18, 2019 
occupancy and data reuse factors and are used to understand and explain the key factors impacting performance. The optimized algorithms are implemented as an open-source software library and we illustrate its use for improving performance of existing or new unstructured-mesh applications.

Keywords: finite volume, finite element, race condition, GPU

\section{Introduction}

Unstructured mesh solvers, particularly applied to the solution of finite difference, finite volume or finite element algorithms, form the basis of numerical simulation applications in a vast area of important scientific domains, from modeling the flow of blood in the body, the flow past an aircraft, to ocean circulation and the simulation of Tsunamis. Significant computational resources are required for the execution of numerical algorithms on these highly detailed (usually three-dimensional) meshes. The solution involves repeatedly iterating over millions of elements (such as mesh edges, nodes, etc.) to reach the desired accuracy or resolution. The key distinguishing feature of these applications is that operations over mesh elements make use of explicit connectivity information between elements to access data defined on neighboring elements. This is in contrast to the use of stencils in structured-mesh applications where the regular geometry of the mesh implicitly provides the connectivity information. As such, iterations over unstructured-meshes lead to highly irregular patterns of data accesses over the mesh, characterized by indirect array accesses. For example, computations over the mesh involve iterating over elements of a set (e.g. faces), performing the same computations, on different data, accessing/modifying data on the set which they operate on (e.g. fluxes defined on the faces), or, using indirections accessing/modifying data defined on other sets (such as coordinate data on connected vertices). These indirect accesses are particularly difficult to 
parallelize when multiple threads may try to modify the same data, leading to data races.

Previous work has utilized one of three approaches for handling data races during parallelization [1, 2]: (1) use coloring where the iteration set is "colored" such that no two iterations of the same color modify the same mesh element indirectly, followed by parallel execution of the iterations with the same color, (2) use large temporary datasets to stage increments without race conditions, and a separate step to gather the increments, or (3) use atomics to handle race conditions. However, the amount of parallelism, and especially the data locality available to be exploited with the above methods have become increasingly limited on modern and emerging massively parallel multi-core and many-core architectures. The performance gains have been limited particularly on many-core processors such as GPUs with thousands of low-power cores, but with modest memory-bandwidth. Thus, reducing data movement and exploiting memory locality during execution is vital on such devices. On GPUs, the first two techniques, coloring or using temporary datasets, end up with poor data locality as one cannot have good data reuse in both reading data as well as writing data without conflicts. The third method, atomics, are much more expensive operations than regular memory transactions and therefore usually lead to low throughput.

In this paper we explore novel data-movement avoiding and locality exploiting algorithms for improving performance of unstructured-mesh applications on GPUs. Identifying that the throughput of memory transactions is the main bottleneck, we demonstrate how superior execution strategies can be obtained by utilizing a combination of techniques from (1) element reordering at threadblock level, (2) use of GPU shared memory as an explicitly managed cache and (3) use of partitioning algorithms for thread-block formation. We show how 
these allow us to maximize data re-use to the higher-bandwidth shared memory, and optimize access patterns to both shared and GPU global memory. More specifically, we make the following contributions:

1. We adopt a caching mechanism on the GPU that loads indirectly accessed elements into GPU shared memory. Then use a two-level "hierarchical coloring" approach to avoid data races, but improve locality over traditional global coloring.

2. We design a reordering algorithm based on graph partitioning that increases data reuse within a thread block, also further increasing shared memory utilization.

3. Finally, we apply the above techniques and optimizations to a number of representative unstructured-mesh applications to investigate performance on modern GPUs, contrasting performance improvements over the stateof-the-art.

We demonstrate how the above locality-exploiting algorithms provide performance improvements of up to $75 \%$ compared to the state-of-the-art on the latest NVIDIA Pascal and Volta GPUs. The algorithms are implemented as an open-source software library [3] which can be used for improving performance of existing or new unstructured-mesh applications.

The rest of the paper is organized as follows: the remainder of Section 1 introduces the basic concepts of unstructured meshes, numerical methods based on them and a discussion on related works, Section 2 describes our optimized algorithms and the motivation leading to the design. Section 3 presents the performance analysis of the algorithms with experimental results. Finally, in Section 4, we present conclusions from this research. 


\subsection{Background}

\subsubsection{Unstructured meshes}

Unstructured meshes can be abstractly viewed as a collection of sets (e.g. nodes, edges, cells, etc.), data defined on these sets (e.g. fluxes, coordinates, velocities), and explicit connectivity information between sets. The connectivity information, declared as mapping tables are required for determining the neighbors of a set element. If we represent sets as consecutive indices from zero to the size of the set, then the mapping between two sets is represented as an array which stores the index of set elements in the second set of the mapping (referred to as the to-set) for every set element of the first set (known as the from-set). For the majority of such applications, the number of to-set elements connected to each from-set element is fixed (e.g. all edges have two vertices). For example consider the mesh illustrated in Figure 1. Part of the mappings from edges to cells for this mesh is detailed in Figure 2. Given such a mapping, we can access the index of those elements that are connected to the current element of the from-set from other sets (the to-sets of the mappings).

The computations on the mesh are declared as a loop over the elements of a set, executing some block of computation on each set element (i.e. an elemental kernel), while accessing data directly on the iteration set or indirectly through a mapping. If a loop over a set only writes to data defined on that set during the elemental kernel, then each iteration of the loop could run in parallel. However, for kernels that indirectly increment data, there may be multiple fromset iterations that update the same to-set element. Such indirect-loops are common in finite volume and finite element applications over unstructuredmeshes: e.g. when updating state variables in cells using fluxes across faces, or when doing matrix assembly. The parallelization of indirect loops are nontrivial as the exact elements leading to data races cannot be determined from 


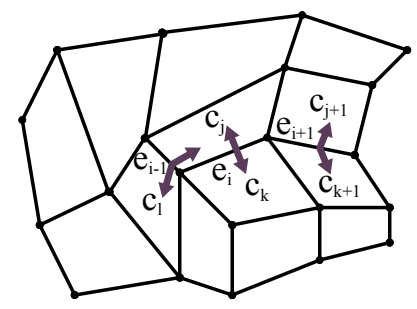

Figure 1: Unstructured mesh, the arrow represents the mapping tells $e_{i}$ is connected to $c_{j}$ and $c_{k}$.

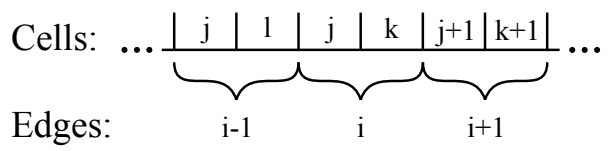

Figure 2: A part of the mapping from edges to cells.

compile time information, given that they are driven by the structure of the mesh in general and the mapping tables in particular, which are read in during run-time.

Some restrictions that apply is also worth noting here. The first is the use of only a single level of mappings. This means that every piece of data that is accessed during an iteration over a set is either defined directly on that set, or is accessed through at most one level of indirection. However, this restriction does not exclude applications using nested indirections, since a mapping table can be created to contain the indexes that we access through multiple mappings. The second restriction is that the result of the operations on the sets are independent from the order of processing the elements of the sets (within machine precision). This restriction enables to exploit the maximum opportunities for parallelization given that the accuracy of the algorithms do not depend on the order of execution. Finally, only mappings with a fixed number of connections (or arity) are considered; such as edges to vertices (where the degree is always 2 ), unlike for a vertices to vertices mapping, where this will vary. The natural formalization of most FEM and FV algorithm uses mappings with fixed number 
of connections.

In spite of the above restrictions, the contributions of the research detailed in this paper is sufficiently general to be applicable to applications that has a computational steps described as an iteration on a set, accessing data on the set and indirectly via a mapping (with a fixed arity). Examples include assembly, certain types of linear algebra algorithms, flux computations, etc.

\subsection{Related Work}

Algorithms defined on unstructured meshes form an important class of applications at many organizations. It is one of the seven dwarfs - common computation-communication patterns or motifs occurring in parallel numerical applications - identified by Colella in 2004 [4]. Discretizations such as finite volumes $(\mathrm{FV})$ or finite elements (FE) often rely on these meshes to deliver high-quality results. Indeed there is a large number of papers detailing such algorithms, and a wide range of commercial, government, and academic research codes (e.g. OpenFOAM [5], Rolls-Royce Hydra [6], FUN3D [7]). All such applications use unstructured meshes in some shape or form, and are often used for large experiments, consisting of millions or even billions of mesh elements. These codes are generally critical to production and consume large portions of high-performance computing systems time. As such, the efficient execution of these applications on the parallel architectures of the day has been and continues to be crucial to the organizations and stake-holders that have invested in them for continued scientific delivery. Over the years, many works have discussed and presented techniques for efficient implementations, initially focusing on traditional CPU architectures [8, 9], then many-core processors such as GPUs (as we discuss below), and even architectures such as FPGAs [10, 11]. Many libraries have also been developed targeting unstructured-mesh solvers, from classical libraries $[12,13]$ to domain specific languages $[14,15,16]$. 
The adoption of GPUs for these kind of computations has already led to considerable speedups over traditional CPU architectures due to the massive parallelism available on GPUs $[17,18,19]$. Other notable works have further looked at improving performance. Remacle et al. [20], explores efficiently solving elliptic problems on unstructured hexahedral meshes on GPUs. They use shared memory for improving data locality, but advanced techniques, such as reordering and partitioning are not utilized. Work done by Castro et al. [21] on implementing path-conservative Roe type high-order finite volume schemes to simulate shallow flows uses auxiliary accumulators to avoid data races while indirectly incrementing. Wu et al. [22] introduce caching using the shared memory with partitioning (clustering), but do not use coloring. Instead they use a duplication method similar to that of LULESH and miniAero, as described below. Fu et al. [23] also create contiguous patches (blocks) in the mesh to be loaded into shared memory, although they partition the nodes (the to-set) but not the elements (the from-set of the mapping). Furthermore, they do not load all data into shared memory, only what is inside the patch. Writing the result back to shared memory is done by a binary search for the column index and atomic adds, which leads to inefficiencies on the GPU.

Parallel to the above work, the US Department of Energy labs have released a set of proxy applications that represent large internal production codes, showing some of the computational and algorithmic challenges to be overcome on novel and emerging architectures Lulesh [24], miniAero [2], BookLeaf [25], MiniFE [26], PENNANT [27]. Out of this suite of codes there are three key approaches to handling data races: (1) allocate large temporary arrays where the intermediate results (i.e. the increments) are placed, avoiding any race conditions, followed by the use of a separate kernel to gather the results, (2) use atomics, (3) use coloring. These all lead to increased warp divergence and high 
data access latencies on GPUs; and the use of the temporary array also leads to more data allocations and movement, further constraining bandwidth.

The research detailed in the present work is based on previous work in [28], where the OP2 library's GPU parallelization use shared memory on GPUs using CUDA for caching with a two level "hierarchical" coloring. However, we demonstrate superior execution strategies on GPUs with reordering of threads and data, to increase data reuse and maximize data locality. Instead of directly porting a specific application to use these techniques we present our methods as general strategies to accelerate unstructured mesh applications, and in particular the indirect increment algorithmic pattern, on GPUs. We have created a classical library as open source software [3] incorporating these optimizations. The library can be used for improving performance of existing or new unstructured-mesh applications.

Most applications of interest for our work implements finite volume algorithms, and low order finite element algorithms, which has a lower computational intensity compared to the number of memory transactions. Thus our optimizations are targeted to avoid data movement, exploiting locality. In contrast high order finite element methods usually have significantly higher computational intensity, where there is a higher number of computations per data element accessed that can hide the cost of the memory access. While our techniques could potentially improve locality, memory bandwidth is less of a concern for such applications.

\section{Parallelization on GPUs}

We begin by outlining the techniques used to effectively optimize unstructured mesh applications on GPUs - some of which are well established and commonly used. We briefly show a naïve solution, then continue with describing various 
improvements found in the literature, and then present our contributions.

\subsection{Traditional parallelization approaches}

On a GPU, groups of threads (warps) run at the same time, in lockstep. As such it is not efficient to execute computations of different length on different threads. Consequently, the usual practice is for each thread to take responsibility for the computation on one element of the set. This can be viewed as running one iteration per thread in the loop over a given set, also known as the iteration set or from-set. This allows the number of computations to be fixed, where the amount of data involved is fixed in the dimension of the mapping and the data arrays.

As mentioned before, care must be taken when writing parallel code to avoid data races when different threads modify the same data. There are three approaches discussed in the literature. The first is to color each thread according to the indirect data it writes, so that no two threads with the same color write the same data, and enforce ordering between colors using synchronization [29]. On the GPU, one would do multiple kernel launches corresponding to the colors, so there is no concurrent writes between threads in the same kernel. We call this the global coloring approach (Figure 3). The disadvantage here is that there is virtually no data reuse: when multiple elements write the same data, they are scheduled for execution in different launches. Since these operations also tend to read data through the same mappings, there is no data reuse in the reads either. Compounding the issue is low cache line utilization where elements of the same color are not neighbors in the mesh, and therefore unlikely to be stored in consecutive memory locations.

The second approach is to serialize the indirect updates by means of locks or atomic additions [30]. This is considerably expensive on the GPU, since the whole warp has to wait at the synchronization step leading to warp divergence. 


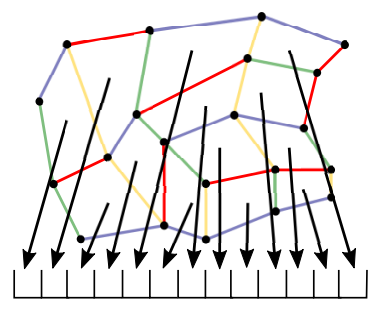

Figure 3: Schematic figure of the global coloring approach. In each kernel launch, the kernels work on edges of the same color. The arrows represent the individual pieces of data loaded indirectly when executing the color red.

The third solution is the use of a large temporary array that stores the results for each thread separately, avoiding race conditions by formulation [1, 2]. However, after the computation finishes, a further kernel is required to gather the results corresponding to one data point. This suffers from the problem of not knowing how many values one thread has to gather, and as a result warps could diverge significantly, and memory access patterns are less than ideal. They can be good either for the write or the read, but not for both. Also, the size of the temporary array is the number of elements multiplied by the dimension of the mapping. As a result, it can be large, for example, in LULESH, it is $8 \times 3 \times$ numElem in our measurements (where numElem is the size of the fromset in LULESH), compared to the array defined on nodes where these values will ultimately end up, which is roughly the same as the number of elements themselves.

\subsubsection{Array-of-Structures (AoS) vs Structure-of-Arrays (SoA)}

Due to the lockstep execution, consecutive threads in a warp read memory at the same time. Therefore, the layout of the data in the memory is an important factor for performance. There are two commonly used layouts [31]: (1) Arrayof-Structures (AoS) layout, where the data associated with one element is in consecutive places in the array (and thus in memory) and (2) Structure-ofArrays (SoA) where the components of elements are stored consecutively e.g. 
the first data component of the elements are in the beginning of the array followed by the second, etc.

Although in most cases the SoA gives better performance on GPUs and better vectorization on CPUs, the AoS layout is still commonly used on $\mathrm{CPU}$ architectures with large caches. In the case of the AoS layout, consecutive threads read data from strided addresses in memory and thus more cache lines are required to satisfy one transaction. This would be compensated by subsequently reading the other components, but may have a negative effect on GPUs due to their small caches. Conversely, with the SoA layout, the threads read data next to each other, which means that the data needed by consecutive threads are most probably in the same cache line resulting in coalesced memory transactions. However, when indirections are involved, these access patterns become more complicated - even with the SoA pattern, consecutive threads may not be reading consecutive values in memory, and therefore cache line utilization degrades. The choice of data layout in unstructured mesh computations is therefore highly non-trivial, as we show later.

\subsection{Shared memory approach}

Considering the three data race avoiding approaches, we see that they all only make use of the GPU global memory. As such one technique to further improve performance is by reducing memory accesses to the GPU global memory. To this end, the OP2 library [28] targets the use of the shared memory on the GPUs. Shared memory is only shared within thread blocks, but has much lower access latency and higher bandwidth than the global memory. The idea is to collect the data required to perform the computations and load it into shared memory. Then, during computation, the indirect accesses are to the shared memory, and the result can also be stored there. After computations by all threads in the block have completed, the contents of the shared memory can be written back to 


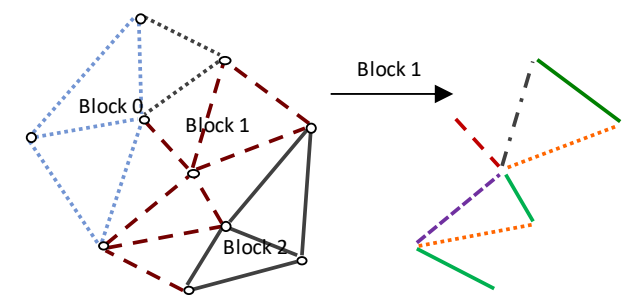

Figure 4: Schematic figure of the two levels of coloring.

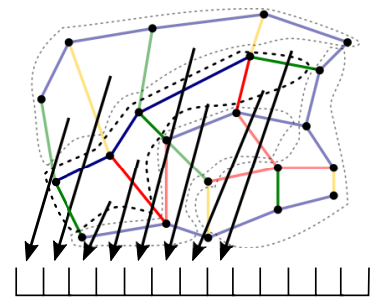

Figure 5: Schematic figure of the hierarchical coloring approach. The thread blocks are circled with dashed lines. The arrows represent the individual data points loaded.

global memory. One immediate advantage of this approach is that the fetching and writing back of data from/to the global memory can be done by the threads independently of the actual threads that will be carrying out the computations on them. Particularly, reading/writing can be done in the order in which the data is laid out in memory, ensuring maximum utilization of cache lines. With the AoS layout, data can be read in contiguous chunks as large as the number of components in the structure.

The use of shared memory of course leads to one additional complication. Writing back the updated patches of shared-memory to GPU global memory may lead to data races. This leads to the use of a two-layered coloring or hierarchical coloring [28] scheme. The two levels of coloring are illustrated in Figure 4, and the associated data accesses are shown in Figure 5. The first level of coloring is to avoid data races when thread blocks write the result back to global memory and the second level is to avoid threads writing their results into shared memory at the same time. 
Algorithm 1 details the steps carried out by a CUDA kernel for executing within this two-level coloring scheme. All indirect data accessed by the block (which is identified during a preprocessing phase) is fetched from global to shared memory to shared memory. As noted in Section 2.1.1, data is usually composed of data points with multiple components (for example, $x-y-z$ coordinates), so this operation consists of two nested loops: (1) an iteration over the data points, and within that, (2) an iteration over the data corresponding to the data point. For the SoA layout, only the outer loop needs to be parallelized, as this will cause parallel read operations to access memory addresses next to each other. For the same reason, if the AoS layout is used, both parallel loops need to be parallelized (i.e. collapsed into one). The data layout in shared memory is best be set to SoA: our measurements showed a consistent degradation in performance when switching to AoS layout, due to the spatial locality described in Section 2.1.1: it leads to fewer bank conflicts.

After the data is loaded into shared memory, each thread executes the main body of the kernel, and outputs are placed into registers. Next, the threads update the result in shared memory with their increments. Finally the updated data is written back to global memory.

One other benefit from using shared memory with hierarchical coloring is the improved data reuse within the block. Each piece of data has to be loaded from global memory only once, but can be used by multiple threads (e.g. data on a shared edge between two triangles). However, the greater the reuse, the more thread colors we have: the number of colors is no less than the number of threads writing the same data. Since the number of synchronizations also grows with the number of thread colors (more precisely, it is the number of colors plus two, one before and one after the computation if the input and the increment are stored separately in shared memory), there is a trade-off between the number of 


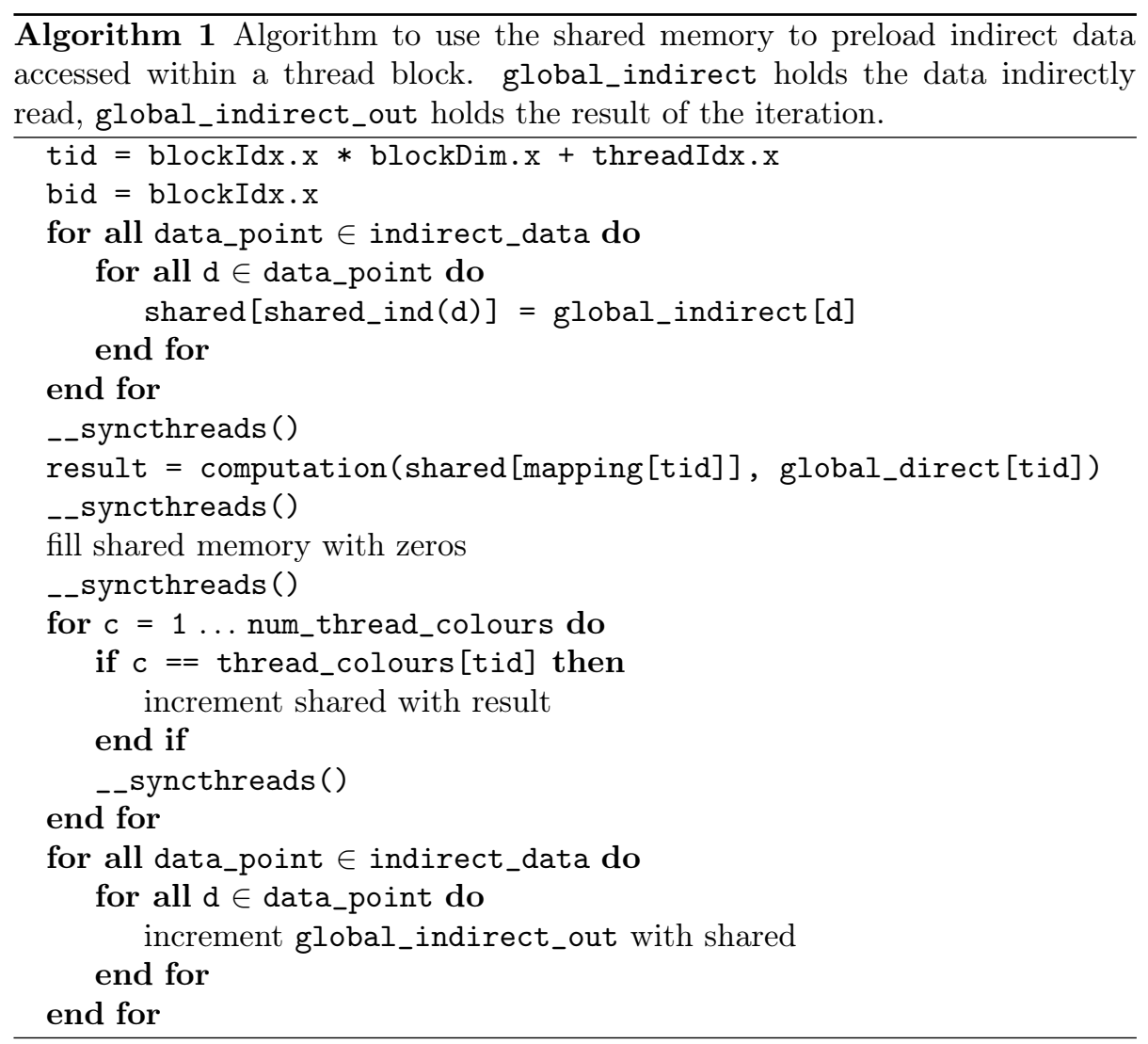


synchronizations and data reuse. Our measurements showed that if the kernel is memory-bound, the greater data reuse leads to increased performance, but the trade-off is non-trivial, as we will demonstrate in Section 3.

\subsection{Increasing data reuse}

Building on the shared-memory with hierarchical coloring, the first contribution of our work attempts to further increase data reuse through reordering of elements. Specifically, reordering of the elements in the from-set (which map directly to the threads), allows us to control how CUDA thread blocks are formed and how much data reuse can be achieved. With the shared-memory approach, the benefit of data reuse is twofold: it decreases the number of global memory transactions and decreases the size of shared memory needed, which leads to greater occupancy. Two different approaches to re-ordering is explored (1) the sparse matrix bandwidth reducing Gibbs-Poole-Stockmeyer algorithm [32] and (2) graph partitioning.

\subsubsection{Gibbs-Poole-Stockmeyer-based reordering}

For serial implementations of computations on graphs (typically on CPUs), the Gibbs-Poole-Stockmeyer algorithm (GPS, [32]) is a heuristic algorithm that increases spatial and temporal locality when traversing the nodes. For example, considering a mesh with edges and nodes, where the edges are the elements of the from-set of the mapping, and the nodes form the to-set, GPS would renumber the nodes and change the order of traversal. The renumbering is done by going through the nodes in a breadth-first manner from two distant starting points, and then renumbers the nodes so that the levels of the resulting spanning trees will constitute contiguous blocks in the new permutation. After

renumbering its points, which by design improves spatial locality, we order the edges of the graph lexicographically, so that consecutive threads (or spatial 


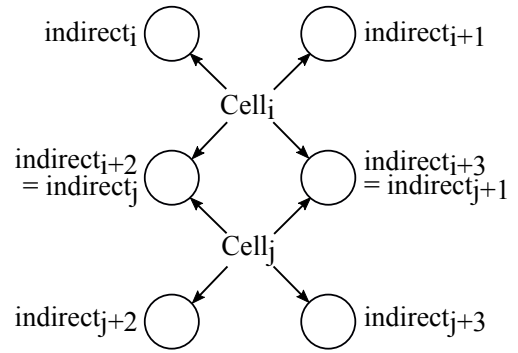

(a)

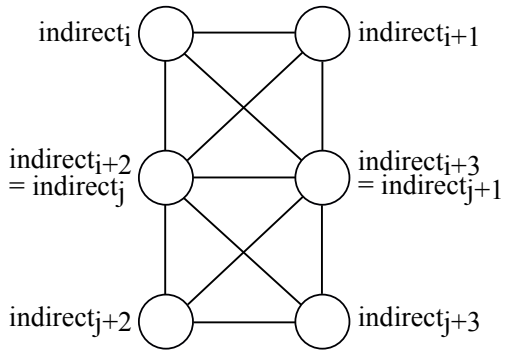

(b)

Figure 6: An example of converting a mesh (shown in (a), with mapping dimension 4) to a graph (on Figure (b)) for the GPS algorithm.

iterations in serial implementations) have a higher chance of accessing the same points, which improves temporal locality (data reuse). The algorithm can be generalized to meshes by transforming each element into a fully connected graph of its points and then taking the union of these. An example of this is shown on Figure 6.

There are several straightforward generalizations to handle multiple sets and mappings (e.g. vertices, edges, cells and their connections). The first is to assume that all the mappings describe a similar topology, so the elements can be reordered based on only one of the mappings (as described above), then reorder the points accessed through the other mappings by, for example, a greedy method. Another approach could be to reorder every data set separately, and then reorder the elements based on the new order of the accessed points, combining the separate data sets (and corresponding mappings) in some way. Since the mappings in the applications we measured are very similar topologically (in fact, except for one of the applications we tested, Airfoil, there is only one mapping in each application), we used the first method. However, the algorithm fails to take into account that on the GPU the threads are grouped into blocks, and data reuse can only realistically be exploited within blocks. This results in blocks that are "pencil-shaped". The next algorithm addresses this limitation. 


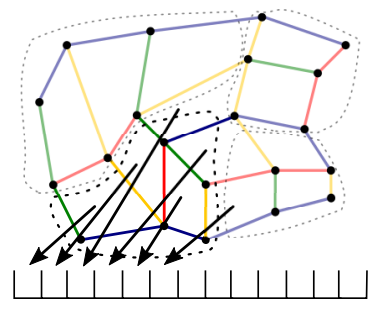

Figure 7: Schematic figure of the hierarchical coloring approach with partitioning. The thread blocks are circled with dashed lines. The arrows represent the individual pieces of data loaded; note that this is less than in Figure 5.

\subsubsection{Partitioning based reordering}

To increase data reuse within a block is equivalent to decreasing data shared between the blocks, more specifically, to decrease the number of times the same data is loaded in different blocks (see Figure 7). With the shared memory approach, data needs to be loaded only once per block. So the task is to partition the elements into blocks of approximately the same size in such a way that when these blocks are assigned to CUDA thread blocks, the common data used (loaded into shared memory) by different blocks is minimized.

Let $G_{M}$ be a graph constructed from the original mapping, where the points are the threads, and there is an edge between them if and only if they access the same data, and let $P_{G_{M}}=\left\{B_{1}, \ldots, B_{n}\right\}$ be a partition of this graph with $n$ blocks. This works even with multiple mappings. If there is a set of blocks $B_{d_{1}}, \ldots, B_{d_{k}}$ that access the same piece of data, then they form a clique in $G_{M}$ in the sense that between any pair of blocks $B_{d_{i}}$ and $B_{d_{j}}$ (where $1 \leq i, j \leq$ $k$ ), there is an edge of $G_{M}$ between $u$ and $v$ such that $u \in B_{d_{i}} \wedge v \in B_{d_{j}}$. Note that the cliques have $0.5 \cdot\left(k^{2}-k\right)$ edges, which is a monotone increasing function in $k$, since $k \geq 1$ (there is at least one block writing each data point, otherwise it is of no relevance). That means that partitioning using the usual objective of minimizing the number of edges between blocks is a good heuristic for maximizing data reuse within the blocks. 
We chose the k-way recursive partitioning algorithm used by the METIS [33] library to partition the graph $G_{M}$. It is a hierarchical partitioning algorithm, where it first coarsens the graph by collapsing nodes, then partitions using the recursive bisection algorithm, and then finally while progressively un-coarsening the graph, locally optimizes the cuts. The algorithm attempts to maintain equal block sizes in the resulting partition, however, it is not always possible because of the underlying algorithm. Since CUDA launches thread blocks with equal size, this must be the maximum of the block sizes in the created partition. Consequently some threads do not do any work, lowering occupancy. One of the tuning parameters for the algorithm is the the load imbalance factor, which can be used to specify the tolerance for this difference. It is called load imbalance because METIS was originally used for distributing computation, ie. load, in a distributed memory system. The Load imbalance factor is defined as $l=$ $n \max _{j}\left\{\operatorname{size}\left(B_{j}\right)\right\}$, where $n$ is the number of blocks and $\operatorname{size}\left(B_{j}\right)$ is the size of the $j$ th block. Due to the local optimization in the un-coarsening phase, it is impractical to set this parameter to 1 (meaning the block sizes must be exactly the same). We found that a tolerance of 1.001 works well in practice for our needs.

We design the block size to be a tuning parameter, which specifies the actual block size of the launched GPU kernels. The number of working threads cannot exceed the block size. To account for this in partitioning, we calculate a new block size $\left(S^{\prime}\right)$ and tolerance $\left(l^{\prime}\right)$ with margins for the imbalance:

$$
\begin{aligned}
S^{\prime} & =\left\lfloor\frac{S}{l}\right\rfloor \\
l^{\prime} & =\frac{S+\epsilon}{S^{\prime}},
\end{aligned}
$$

where $S$ is the original block size, $l$ is the original load imbalance parameter 
and $\epsilon$ is an empirical tuning parameter to create as large blocks (within the limit) as possible. This support for a variable number of working threads (ie. to determine if the current thread should do any actual computation) also incurs a slight overhead of having to load the start and end index for each block. We found this overhead to be minimal in practice.

Due to the way loads and stores work on the GPU, what actually affects performance is not the number of data points accessed, but rather the number of cache lines (of size 32 bytes on the hardware used) that are accessed. A simple heuristic reordering of data points is used to account for this.

The idea is to group data points together (in a contiguous chunk of memory) that are read/written by the same set of blocks: this makes them more likely to be loaded in the same cache line. This is even more important when more blocks access the same group of data points (set elements on the boundary), since then inefficiencies will worsen performance for each of these blocks. As a simple heuristic, we group data points with the same number of blocks that access them together (by sorting), and within these groups, we sort by the indices of the accessing blocks lexicographically.

It must be noted that GPS and METIS were developed for distributing workloads on computing clusters that typically have much larger block size to total size ratio. This also caused the reordering (partitioning) phase, running on a single CPU core, to be quite long: for example, the GPS reordering took 5 seconds, the partitioning 60-90 seconds and the hierarchical coloring 5-9 seconds (depending on the block size and whether the mesh was partitioned) on the mesh used by Airfoil. However, this is a one-off cost: the reordering can be reused many times later. Further improvement could be achieved by using the parallel versions of the METIS library: ParMETIS[34] and the alpha version mt-METIS[35] libraries. Partitioning algorithms targeting specifically small 
partition sizes required for CUDA thread blocks, and improving its performance were out of scope for this work.

\subsection{Further optimizations}

There are a number of further optimizations we introduced to improve performance. We can increase the number of loads or stores in flight to/from global memory by using CUDA's built-in vector types (float2, float4 and double2). This way, each thread will load multiple consecutive values from memory during a single transaction. This is useful to increase the efficiently of loads that are already coalesced.

When updating the shared memory with increments, the threads within a block can be sorted by their color. Then threads with the same color will be next to each other, so warps will have fewer threads of different colors. This results in reduced warp divergence on average.

Marking pointers to data on the GPU with __restrict_-_ and const where applicable enables the compiler to apply further reordering optimizations, which it would not have deemed safe to do otherwise. _-_restrict_-_ instructs the compiler that the pointers do not alias one another, ie. do not point to the same memory space. The const enables the compiler to place the data in texture cache that has lower latency than the global memory.

\section{Performance}

For the remainder of the paper, we present a detailed investigation into the performance implications of the locality-exploiting optimizations on a number of representative unstructured-mesh applications. The applications are all well established in literature and are representative of several domains that make use of unstructured mesh codes. Our aim is to present the performance of the state- 


\begin{tabular}{lcc}
\hline & P100 (GP100) & V100 (GV100) \\
\hline \hline Streaming Multiprocessors (SM) & 56 & 80 \\
\hline Max Thread Block Size & 1024 & 1024 \\
\hline Max Warps / Multiprocessor & 64 & 64 \\
\hline Max Threads / Multiprocessor & 2048 & 2048 \\
\hline Max Thread Blocks / Multiprocessor & 32 & 32 \\
\hline Max Registers / Thread & 255 & 255 \\
\hline Shared Memory Size / SM & $64 \mathrm{~KB}$ & $96 \mathrm{~KB}$ \\
\hline Max 32 - bit Registers / SM & 65536 & 65536 \\
\hline Memory Size & $16 \mathrm{~GB}$ & $16 \mathrm{~GB}$ \\
\hline L2 Cache Size & $4096 \mathrm{~KB}$ & $6144 \mathrm{~KB}$ \\
\hline Peak Stream Bandwidth & $495 \mathrm{~GB} / \mathrm{s}$ & $742 \mathrm{~GB} / \mathrm{s}$ \\
\hline
\end{tabular}

Table 1: Important informations about the NVIDIA Tesla P100 and V100 GPUs [36, 37]

of-the-art on GPUs with these applications and then contrast the performance gained with our contributions.

\subsection{Experimental setup}

The GPU systems used in performance analysis is detailed in Table 1. These consist of two of NVIDIAs latest high-performance computing Tesla GPUs, namely the P100 and V100. The GPUs are hosted in a server with an Intel Xeon CPU E5-1660 (3.20GHz base frequency, 1 socket with 8 cores) running Ubuntu 16.04. The nvcc compiler with CUDA version 9.0 (V9.0.176) is used.

Both runtime performance as well as low-level metrics on the GPUs such as achieved bandwidth and occupancy is utilized to understand the bottlenecks affecting performance. There are three key factors of interest: (1) the coloring approach (global or hierarchical) giving the best performance, (2) method of data reordering (no-reordering, GPS-based, or graph partitioning-based) and (3) data layout (AoS or SoA). All combinations of these are evaluated and compared to each other and a state-of-the-art reference implementation.

When comparing performance of different versions, we use the achieved bandwidth as the key performance metric. Emphasis on bandwidth is justified 
given that all of the test applications, as we demonstrate, are memory-bound. Achieved bandwidth is calculated by the formula:

$$
\frac{\sum_{d} w_{d} S_{d}}{T} \cdot I
$$

where $d$ iterates over the datasets, $w_{d}$ is 2 if the data is read and written, 1 otherwise, $S_{d}$ is the size of the dataset (in bytes), $T$ is the overall runtime of the kernel and $I$ is the number of iterations. A number of additional metrics were also collected, these include:

- data reuse factor (the average number of times an indirectly accessed data point is accessed),

- the number of read/write transactions from/to global memory, which is closely related to the data reuse factor but is affected by memory access patterns, and therefore cache line utilization,

- the occupancy reflecting the number of threads resident on the SM versus the maximum - the higher this is, the better chance of hiding the latency of compute/memory operations and synchronization

- the percentage of stalls occurring because of data requests, execution dependencies, or synchronization,

- the number of block colors; the higher it is, the less work in a single kernel launch, which tends to lead to lower utilization of the GPU,

- the number of thread colors; the higher this is the more synchronizations are required to apply the increments in shared memory — but also strongly correlates with data reuse,

- warp execution efficiency (ratio of the average active threads per warp to the maximum number of threads per warp). 
Studying runtime performance and the above metrics enables us to understand and explain why certain variants are better than others.

\subsubsection{Airfoil}

Airfoil, implemented using the OP2 DSL [28] is the smallest, best understood and most thoroughly studied among the applications we explored. It is representative of large industrial Finite Volume CFD applications and implements a non-linear 2D inviscid airfoil code using an unstructured grid. A finite-volume discretization is used to solve the 2D Euler equations with a scalar numerical dissipation. The algorithm iterates towards the steady state solution, in each iteration using a control volume approach, meaning the change in the mass of a cell is equal to the net flux along the four edges of the cell, which requires indirect connections between cells and edges. Two versions of the code exists, one implemented with OP2's C/C ++ API and the other using OP2's Fortran API $[15,38]$.

The application consists of five parallel loops in total: save_soln, adt_calc, res_calc, bres_calc and update. Here we focus on res_calc, as it has indirect increments and about $70 \%$ of the total runtime of the application is spent in this parallel loop on GPUs when using a global coloring. The loop contains both indirect reads and writes. It iterates through edges (i.e. the from-set), and computes the flux through edges using data accessed indirectly on the two cells adjacent to each edge. The res_calc loop is called 2000 times during the execution of the application and performs about 100 floating-point operations per mesh edge. In each iteration, it reads 5 and increments 4 double values from

each of the 2 indirectly accessed cells, and reads 2 double values from each of the 2 indirectly accessed nodes.

Table 2 show the effect of various optimizations on the Airfoil application's res_calc kernel, during the execution on a mesh with 2.8 million cells. 


\begin{tabular}{|c|c|c|c|c|c|c|c|c|}
\hline Coloring & \multicolumn{3}{|c|}{ Global } & \multicolumn{4}{|c|}{ Hierarchical } & \multirow{3}{*}{$\begin{array}{c}\begin{array}{c}\text { Original } \\
\text { Hierarchical }\end{array} \\
\text { none } \\
\text { SOA }\end{array}$} \\
\hline Reordering & none & \multirow{2}{*}{$\begin{array}{l}\text { GPS } \\
\text { SOA }\end{array}$} & \multirow{2}{*}{$\frac{\text { partition }}{\text { SOA }}$} & \multicolumn{2}{|c|}{ none } & \multicolumn{2}{|c|}{ partition } & \\
\hline Data layout & AOS $\quad$ SOA & & & AOS & SOA & AOS & SOA & \\
\hline Bandwidth (GB/s) & 72 & 106 & 66 & 211 & 215 & 228 & 239 & 233 \\
\hline Runtime (ms) & 6.12 & 4.15 & 6.64 & 2.07 & 2.03 & 1.92 & 1.83 & 1.91 \\
\hline $\begin{array}{r}\text { Achieved } \\
\text { Occupancy }\end{array}$ & 0.45 & 0.45 & 0.45 & 0.44 & 0.43 & 0.44 & 0.43 & 0.42 \\
\hline $\begin{array}{r}\text { Global Memory } \\
\text { Read Transactions }\end{array}$ & $52424 \mathrm{k} \quad 45781 \mathrm{k}$ & $41246 \mathrm{k}$ & $66775 \mathrm{k}$ & $21142 \mathrm{k}$ & $21275 \mathrm{k}$ & $13885 \mathrm{k}$ & $14325 \mathrm{k}$ & $21866 \mathrm{k}$ \\
\hline $\begin{array}{r}\text { Global Memory } \\
\text { Write Transactions }\end{array}$ & $14007 \mathrm{k} \quad 14737 \mathrm{k}$ & $13773 \mathrm{k}$ & $20733 \mathrm{k}$ & $5807 \mathrm{k}$ & $5871 \mathrm{k}$ & $3429 \mathrm{k}$ & $3628 \mathrm{k}$ & $6384 \mathrm{k}$ \\
\hline $\begin{array}{r}\text { Number of (Block) } \\
\text { Colours }\end{array}$ & 5 & 5 & 7 & 4 & 4 & 8 & 8 & 5 \\
\hline $\begin{array}{r}\text { Number of Thread } \\
\text { Colours }\end{array}$ & - & - & - & 3 & 3 & 4 & 4 & 3 \\
\hline Reuse Factor & - & - & - & 2 & 2 & 3.6 & 3.6 & 2 \\
\hline $\begin{array}{r}\text { Issue Stall Reasons } \\
\text { (Synchronization) }\end{array}$ & - & - & - & $11 \%$ & $10 \%$ & $15 \%$ & $14 \%$ & $14 \%$ \\
\hline $\begin{array}{r}\text { Issue Stall Reasons } \\
\text { (Data Request) }\end{array}$ & - & - & - & $69 \%$ & $70 \%$ & $62 \%$ & $64 \%$ & $55 \%$ \\
\hline Block Size & \multicolumn{7}{|c|}{480} & 128 \\
\hline
\end{tabular}

Table 2: Low-level performance metrics of Airfoil's res_calc kernel - global coloring vs hierarchical coloring (2.8 million mesh cells). The last column details the measured performance of the original code.

Global coloring: We see that using the SoA layout improves performance.

As discussed in Section 2.1.1, with SoA threads in a warp access data addresses that are near each other. The improvement can also be seen in the number of global memory read transactions as it is roughly $87 \%$ of that with AoS layout. Adding the GPS renumbering improves performance further by placing data points that are accessed in consecutive threads close to each other. Now there is a $19 \%$ reduction in global read transactions compared to the baseline AoS. Given that the partition based reordering is primarily intended for the hierarchical coloring, it does not improve on the Global coloring. The reason being that partitioning groups threads that access the same data together, while the global coloring puts them into different kernel launches, eliminating any chance for spatial reuse.

Hierarchical coloring: The key goal of this strategy is to better exploit data reuse by using the GPU shared memory. The effectiveness of the approach show immediately due to the significant reduction in the number of global transactions in Table 2. At block size 480, there is roughly a $60 \%$ decrease in global read 
and write transactions, leading to three times the performance. Throughput for different block sizes is shown in Figure 8.

We also see that reordering using partitioning is indeed more effective. With a block size of 448 , data reuse increases from 2 on the reference version, to 3.6 , leading to the $19 \%$ performance gain over the version without reordering (AoS layout). This is also consistent with the number of global transactions: there is a $35 \%$ decrease in the number of reads and $41 \%$ decrease in the number of writes, and a decrease in the percentage of stalls occurring because of data requests: $61 \%$ with partitioning, $68 \%$ without.

With the increased reuse, the number of thread colors is also larger (4 versus 2.2 ) and this leads to more synchronization. With reordering, $14 \%$ of the stalls were caused by synchronization, up from $9 \%$. This is further illustrated by Figure 9 that shows the relative speedup compared to the original OP2 version (its low-level metrics are detailed in the final column of Table 2). In this case, the original version also used the shared memory approach, so the performance gains are caused by the reordering. In the original version (hierarchical coloring, SOA layout) $56 \%$ of the total time is spent in res_calc. The best original version used the SOA data layout, with reordering we achieved $19 \%$ speedup on res_calc with AOS layout. However with AOS layout we lose performance in direct kernels, therefore regarding the whole application one can reach better performance with the SOA layout. The best performing setting of airfoil reached about $3.3 \%$ speedup on the whole application. The useful bandwidth of the best performing version of res_calc (our implementation) reached $55 \%$ of the peak stream bandwidth of the P100 GPU.

Similar results were obtained on the newer Volta GPUs (V100) as illustrated in Figure 10). The absolute value of the bandwidths are (understandably) higher. On the V100, AOS achieved $28 \%$ speedup in the kernel compared to the 

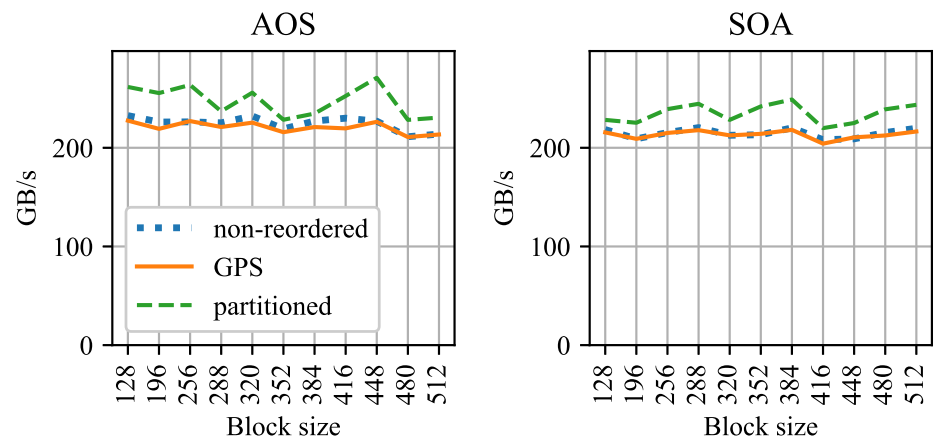

Figure 8: Airfoil's res_calc bandwidth on a dataset with 2880000 cells with hierarchical coloring.

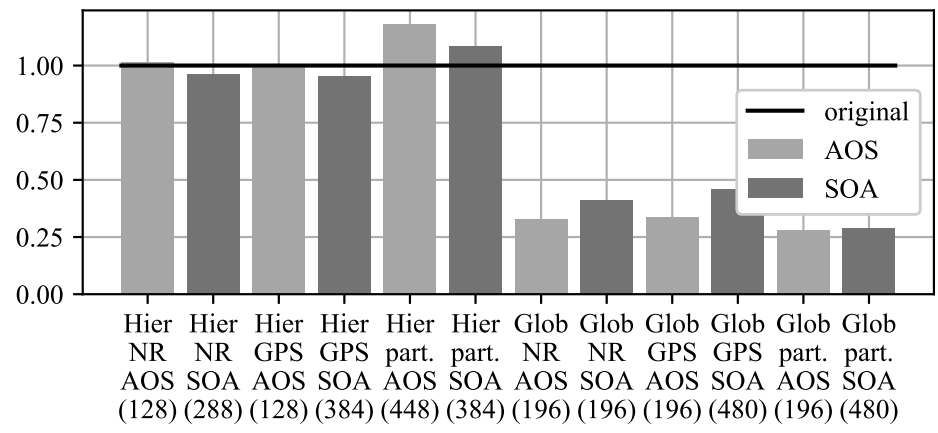

Figure 9: Airfoil's res_calc kernel speedup compared to the original code (on a mesh with 2800000 cells. The block sizes are shown in parentheses, the reordering algorithms are: the original reordering (NR), GPS reordering and partitioning (part.)
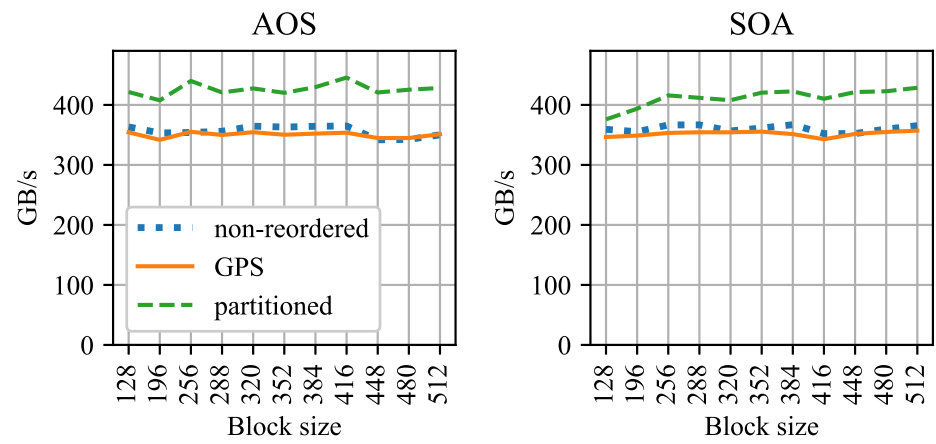

Figure 10: Airfoil's res_calc bandwidth on a dataset with 2880000 cells with hierarchical colouring, on Volta architecture. 
original code with $446 \mathrm{~GB} / \mathrm{s}$ bandwidth ( $60 \%$ of the peak bandwidth), but the it still lacks behind SOA regarding the whole application. The best SOA version achieved $21 \%$ speedup. Since res_calc takes around $54 \%$ of the total run time this speedup lead to $11 \%$ performance increase on the whole application.

\subsubsection{Volna}

The next application we explore here, Volna, is in fact a production/research code for shallow water simulation capable of handling the complete life-cycle of a tsunami (generation, propagation and run-up along the coast) [39]. The simulation algorithm works on unstructured triangular meshes and uses the finite volume method. Volna is written in $\mathrm{C} / \mathrm{C}++$ and is converted to use the OP2 library [40]. Volna spends most time in three kernels: computeFluxes, SpaceDiscretization and Numericalfluxes. Out of these, we focus on the SpaceDiscretization kernel that iterates on edges accessing data indirectly on cells, $60 \%$ of total execution time is spent in this kernel. In each iteration, SpaceDiscretization reads 1 and increments 4 float values from each of the 2 indirectly accessed cells, and reads 7 float and 1 integer values directly. A notable difference in Volna is that the execution with single precision is adequate for solution accuracy. As such we benchmark it with single precision floatingpoint mathematics, on a mesh containing 2.4 million triangular cells, simulating a tsunami run-up to the US pacific coast.

Figure 11 and Table 3 details the performance metrics observed for the SpaceDiscretization kernel in Volna. We concentrate solely on the hierarchical coloring variants given their superior performance to global coloring. The reordering by partitioning again improves performance. It increases reuse from 1.5 to 2.8 and decreases the number of global transactions by $18 \%$ for reads and $37 \%$ for writes. The larger reduction in writes can be explained by the fact that the calculation only reads data defined on the iteration set directly. 

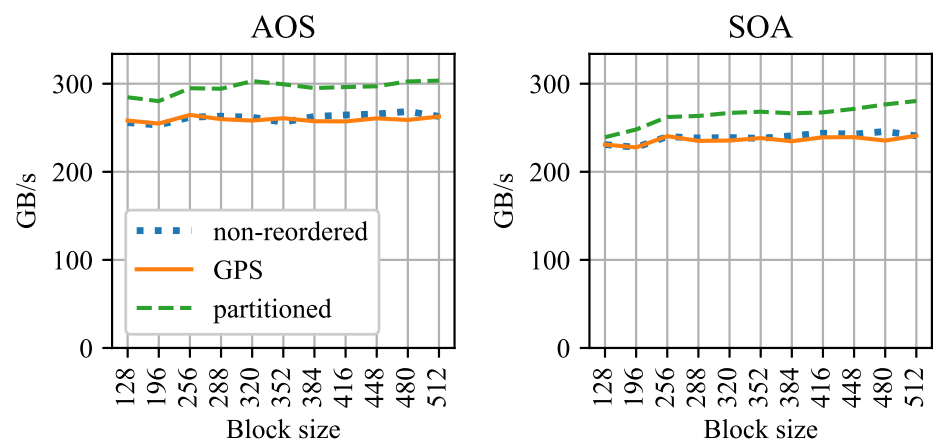

Figure 11: Volna's SpaceDiscretization kernel bandwidth on a mesh with 3589735 edges using hierarchical coloring.

Again, recall that the AoS version uses adjacent threads to load adjacent components of data points. Additionally, given the use of single precision values for Volna, one thread loads 4 single precision values into shared memory using the built-in vector type float4. Consequently, more data is transferred at the same time, providing a $2 \%$ and $4 \%$ reduction in global memory transfers for reads and writes, respectively. This leads to performance improvements of $292 \mathrm{~GB} / \mathrm{s}$ versus $268 \mathrm{~GB} / \mathrm{s}$.

Low register counts (28-32) and single-precision data types also resulted in achieving a higher occupancy on the GPU compared to Airfoil. This, we believe explains why performance appears to be independent of the block size as shown in the Figure 11.

We also see that using partitioning does not increase the number of thread colors significantly, as we observed on Airfoil. The increase of colors are from 3 to 4 . As such the overall synchronization overhead is also smaller. The percentage of stalls caused by synchronization increases from $12 \%$ to just $15 \%$. Of course, with high occupancy, the latency caused by synchronization can be better hidden by running warps from other blocks.

As can be seen in Figure 12, the locality exploiting optimizations make the 


\begin{tabular}{|r|cc|c|c||c|}
\hline Reordering & \multicolumn{2}{|c|}{ none } & \multicolumn{2}{c||}{ partition } & original \\
\hline Data layout & AOS & SOA & AOS & SOA & SOA \\
\hline Bandwidth (GB/s) & 133 & 120 & 146 & 134 & 119 \\
Runtime (ms) & 0.87 & 0.95 & 0.77 & 0.85 & 0.93 \\
Achieved Occupancy & 0.82 & 0.81 & 0.80 & 0.80 & 0.68 \\
Global Memory Read Transactions & $9114 \mathrm{k}$ & $9166 \mathrm{k}$ & $7493 \mathrm{k}$ & $7617 \mathrm{k}$ & $9504 \mathrm{k}$ \\
Global Memory Write Transactions & $2438 \mathrm{k}$ & $2512 \mathrm{k}$ & $1542 \mathrm{k}$ & $1640 \mathrm{k}$ & $2809 \mathrm{k}$ \\
Number of Block Colours & 5 & 5 & 9 & 9 & 6 \\
Number of Thread Colours & 3 & 3 & 4 & 4 & 3 \\
Reuse factor & 1.5 & 1.5 & 2.8 & 2.8 & 1.48 \\
Issue Stall Reasons (Synchronization) & $11 \%$ & $12 \%$ & $15 \%$ & $15 \%$ & $14 \%$ \\
Issue Stall Reasons (Data Request) & $51 \%$ & $50 \%$ & $46 \%$ & $46 \%$ & $47 \%$ \\
Average cache lines/block & 300 & 307 & 165 & 184 & - \\
Warp Execution Efficiency & $98 \%$ & $98 \%$ & $97 \%$ & $97 \%$ & $65 \%$ \\
\hline Block size & \multicolumn{5}{|c||}{307} \\
\hline
\end{tabular}

Table 3: Low-level performance metrics of Volna's SpaceDiscretization kernel - hierarchical coloring. The last column details the measured performance of the original code.

kernel $20 \%$ faster than the original OP2 version. Again the best performance for SpaceDiscretization was reached with hte AOS layout, which is not optimal for the whole application - overall the best total performance can be reached with SOA layout. Notably, ComputeFluxes also benefits significantly from the locality optimization, since this kernel also iterates on the edges and read data from cells; we experienced $5 \%$ speed increase in ComputeFluxes. The increased speed of the two kernels results in a $13 \%$ increase for the whole application. The useful bandwidth also increases and reached $30 \%$ of the peak stream bandwidth of the P100 GPU.

On the V100, the performance of the kernel was $462 \mathrm{~GB} / \mathrm{s}$ ( $62 \%$ of the peak bandwidth) with $18 \%$ (AOS) speedup compared to the original implementation. Running the whole application with the reordered mesh, SOA achieved $11 \%$ speedup on SpaceDiscretization while improving ComputeFluxes with $10 \%$ as well, leading to a $8 \%$ speedup on the whole application compared to the original implementation. 


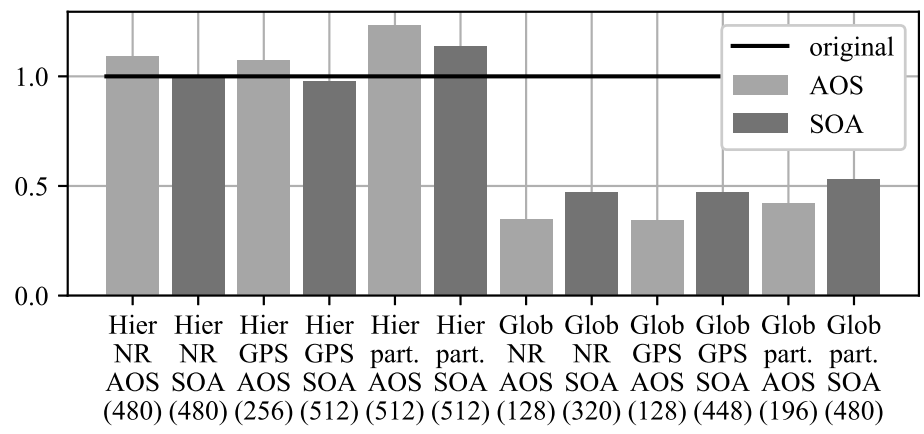

Figure 12: Volna's SpaceDiscretization kernel speedup compared to the original code, done on a mesh with 3589735 edges. The block sizes are shown in parentheses, the reordering algorithms are: the original reordering (NR), GPS reordering and partitioning (part.)

\subsubsection{BookLeaf}

The third application we explore is BookLeaf. It is a $2 \mathrm{D}$ unstructured mesh Lagrangian hydrodynamics application from the UK Mini-App Consortium [41]. It uses a low order finite element method with an arbitrary Lagrangian-Eulerian method. BookLeaf is written entirely in Fortran 90 and has been ported to use the OP2 API and library. BookLeaf has a large number of kernels with different access patterns such as indirect increments similar to increments inside res_calc in Airfoil. For benchmarking we used the SOD test case with a mesh of 4 million cells. The top time consuming kernel with indirect increments is getacc_scatter, which iterates on cells while incrementing data indirectly on vertices, $6 \%$ of total execution time is spent in this kernel. This kernel reads 17 double values directly, and increments 4 double values on each of the 4 indirectly accessed nodes in each iteration.

Runtime performance of BookLeaf and specifically the low-level performance of the getacc_scatter kernel are detailed in Figures 13 and 14. Again we see benefits from partitioning. The register count and occupancy are also similar to those with Airfoil (64 registers, achieving occupancy around 40\%), this now leads 

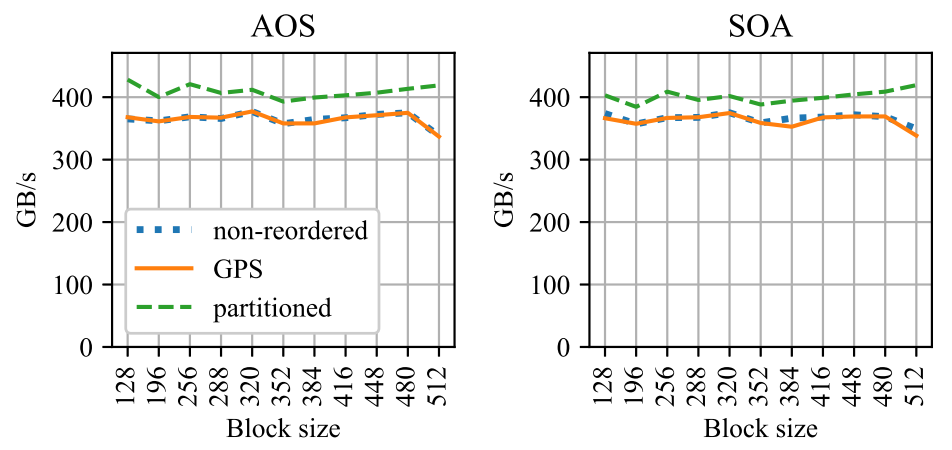

Figure 13: Bookleaf's getacc_scatter kernel bandwidth on a mesh with 4000000 edges using hierarchical colouring.

to the variations in performance for different block sizes. With partitioning, the number of thread colors increases from 2 to 5 , this leads to increased stalls from synchronizations: from $9 \%$ to $20 \%$, while the reuse factor increases (from 2 to 3.5). This is comparable to that of Airfoil, and explains the smaller increase in performance (only 15\%, compared to the $19 \%$ increase in Airfoil). The higher data reuse leads to $14 \%$ and $41 \%$ decrease of the number of global transactions, for reads and writes, respectively. Such a large difference between reads and writes is also due to getacc_scatter having no indirect reads, similar to like SpaceDiscretization in Volna.

The best performance we achieved with getacc_scatter $(1.15 \times$ speedup $)$ results in about $1 \%$ performance increase for the the whole application. The useful bandwidth also increased and reached $83 \%$ of the peak stream bandwidth of the P100 GPU. On the V100, the performance of the kernel was $631 \mathrm{~GB} / \mathrm{s}$ ( $85 \%$ of the peak bandwidth) which results in $0.5 \%$ runtime speedup on the whole application compared to the original. 


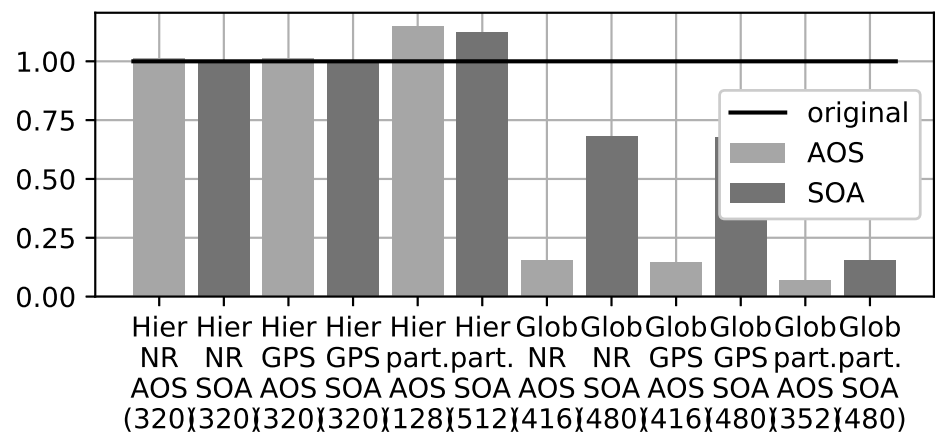

Figure 14: Bookleaf's getacc_scatter kernel speedup compared to the original code, done on a mesh with 4000000 edges. The block sizes are shown in parentheses, the reordering algorithms are: the original reordering (NR), GPS reordering and partitioning (part.)

\subsubsection{LULESH}

LULESH (Livermore Unstructured Lagrangian Explicit Shock Hydrodynamics [24]) application represents a typical hydrocode representing the Shock Hydrodynamics Challenge Problem that was originally defined and implemented by Lawrence Livermore National Lab as one of five challenge problems in the DARPA UHPC program. It has since become a widely studied proxy application in DOE co-design efforts for exascale.

LULESH is a highly simplified application, hard-coded to only solve a simple Sedov blast problem that has an analytic solution [1] - but represents the numerical algorithms, data motion, and programming style typical in scientific $\mathrm{C} / \mathrm{C}++$ based applications at the Lawrence Livermore National Laboratory. LULESH approximates the hydrodynamics equations discretely by partitioning the spatial problem domain into a collection of volumetric elements defined by a mesh. The mesh itself is structured (and generated in the code), but the algorithm doesn't take this into account and accesses the data through an eight-dimensional mapping for the hex8 (brick) elements.

We explore the IntegrateStressForElems kernel that calculates the forces 
in the nodes. Iterating over cells, it reads 3 double values from each of the 8 indirectly accessed nodes, increments 3 double values for each node, reads 3 double values directly and writes 1 double value directly. In our measurements, we used a mesh with 4913000 cells and 5000211 nodes. The original CUDA version of the code contracted this kernel with CalcFBHourglassForceForElems; the only modifications we applied to this code for our tests was to remove these parts from the kernel.

The IntegrateStressForElems kernel uses a mapping with 8 neighbors for the brick elements (compared to the 2-4 as in the case of the previous application kernels). As a result, the number of block colors is quite high: 8, 16 and 24 in the global coloring versions (for the different reorderings), and 4, 5 and 15 in the hierarchical coloring versions. The number of thread colors was also quite high: 4 in the non-reordered (4.5 in the GPS) and 11.6 in the partitioned version. The non-reordered and GPS versions yield blocks that have "pencil shape", thus requiring fewer thread colors, whereas the partitioned version yields more cubical shaped blocks, leading to the higher number of thread colors. This is a much larger increase compared to the previous applications (Table 4). At the same time of course, data reuse is higher compared to $2 \mathrm{D}$ applications between 2.6 and 4.8 .

The other aspect in which LULESH is differs is that it uses a high amount of registers (96), which significantly decreases occupancy: with block size 320 , the AoS version achieved $15 \%$ and the SoA version achieved around $30 \%$. Because of these two reasons, the synchronization overhead (39\% stalls were from synchronization on the partitioned mesh) couldn't be hidden: there were no warps from other blocks to be scheduled in place of the stalled ones because there was only one block running on each multiprocessor. The difference in achieved occupancy also means that the SoA version with two blocks per multiprocessor 

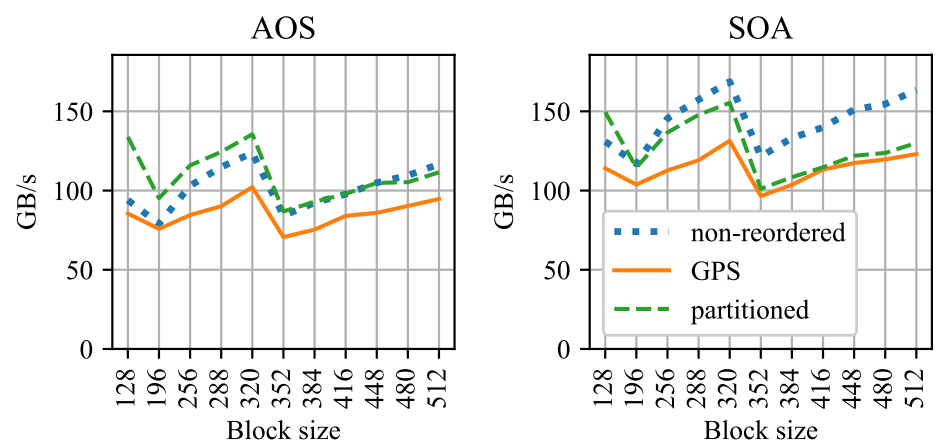

Figure 15: LULESH's IntegrateStressForElems kernel bandwidth on a mesh with 4913000 cells using hierarchical colouring.

gives better performance.

The original implementation used either atomics or helper arrays as a means of avoiding data races. As shown in Figure 16, the hierarchical coloring algorithm performs significantly better (giving a $49 \%$ speedup) than the original two-step implementation (and also uses much less memory), but it is worse than the original atomics implementation by $17 \%$.

In the original application version $37 \%$ out of the total time is spent in the IntagrateStressForElems kernel, therefore the achieved $49 \%$ speedup on the kernel over the two-step implementation gives about $11 \%$ (for our best result) or $8 \%$ (for our partitioned version) speedup on the whole application. This was measured by reading back the reordered mesh in the original code for all kernels. The useful bandwidth in case of the best version of IntegrateStressForElems reached $35 \%$ of the peak stream bandwidth on the P100 GPU.

On the V100, we achieved $51 \%$ speedup for this kernel compared to the original two-step implementation with $270 \mathrm{~GB} / \mathrm{s}$ bandwidth $(36 \%$ of the peak bandwidth). In the original version $38 \%$ of the total time is spent in IntegrateStressForElems, therefore this achieved kernel speedup results in about $16 \%$ speedup on the whole application. Again, the atomics version performed best with a bandwidth 


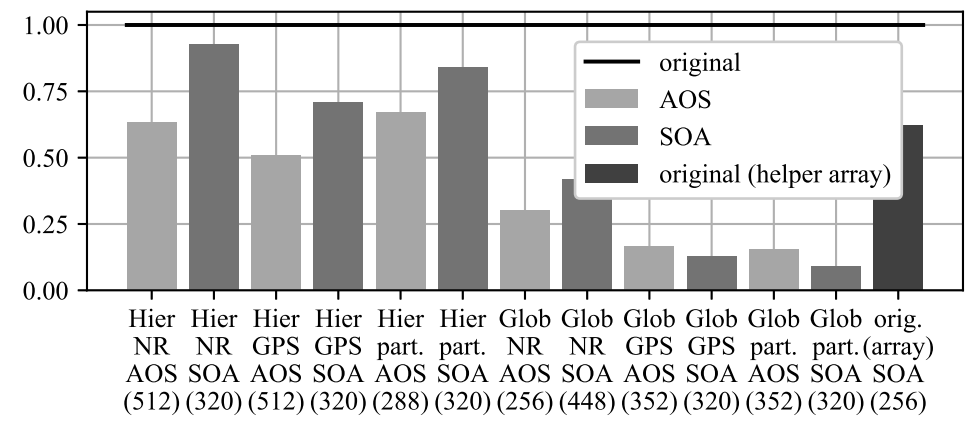

Figure 16: LULESH's IntegrateStressForElems kernel speedup compared to the original (gathering) code, done on a mesh with 4913000 cells. The block sizes are shown in parentheses, the reordering algorithms are: the original reordering (NR), GPS reordering and partitioning (part.) The last bar shows the relative performance of the original code with the helper array approach.

of $561 \mathrm{~GB} / \mathrm{s}$ ( $76 \%$ of peak bandwidth), two times faster than our version.

\subsection{5. miniAero}

The final application we explore is miniAero [2], which is a mini-application from the Mantevo suite [42]. MiniAero is an explicit (4th order Runge-Kutta) unstructured finite volume code that solves the compressible Navier-Stokes equations. Both inviscid and viscous terms are included. The viscous terms can be optionally included or excluded. For miniAero, meshes are created within the code and are simple 3D hex8 meshes. These meshes are generated on the CPU and then moved to the GPU. While the meshes generated in code are structured, the code itself uses unstructured mesh data structures and access patterns. This mini-application uses the Kokkos library [43].

For miniAero we study the compute_face_flux kernel that computes the flux contributions of the faces and increments it with the appropriate cell flux values. The kernel iterates over the faces of the mesh, and accesses the cells indirectly. In each iteration, it reads 28 and increments 5 double values from each of the 2 indirectly accessed cells, and reads 12 double values directly. The 


\begin{tabular}{r|cc|c|c||c|}
\hline Reordering & \multicolumn{2}{|c|}{ none } & \multicolumn{2}{c|}{ partition } & original \\
\hline Data layout & AOS & SOA & AOS & SOA & SOA \\
\hline Bandwidth (GB/s) & 80 & 172 & 83 & 157 & 97 \\
Runtime (ms) & 8.48 & 3.90 & 8.10 & 4.31 & 6.98 \\
Achieved Occupancy & 0.15 & 0.29 & 0.15 & 0.30 & 0.24 \\
Global Memory Read Transactions (total) & $33546 \mathrm{k}$ & $35395 \mathrm{k}$ & $22883 \mathrm{k}$ & $25006 \mathrm{k}$ & $16072 \mathrm{k}$ \\
Global Memory Write Transactions (total) & $12674 \mathrm{k}$ & $12706 \mathrm{k}$ & $8052 \mathrm{k}$ & $8703 \mathrm{k}$ & $32689 \mathrm{k}$ \\
Number of Block Colours & 4 & 4 & 15 & 15 & - \\
Number of Thread Colours & 4 & 4 & 9.8 & 9.8 & - \\
Reuse Factor & 2.6 & 2.6 & 4.8 & 4.8 & - \\
Issue Stall Reasons (Synchronization) & $13 \%$ & $19 \%$ & $36 \%$ & $39 \%$ & $0 \%$ \\
Issue Stall Reasons (Data Request) & $63 \%$ & $56 \%$ & $38 \%$ & $34 \%$ & $26 \%$ \\
Average Cache Lines/Block & 744 & 747 & 427 & 474 & - \\
Warp Execution Efficiency & $98 \%$ & $98 \%$ & $94 \%$ & $93 \%$ & $100 \%$ \\
\hline Block size & \multicolumn{4}{|c|}{320} & 64 \\
\hline
\end{tabular}

Table 4: Low-level performance metrics of LULESH's IntegrateStressForElems kernel - hierarchical coloring. The last column details the measured performance of the original kernel.

original code, depending on a compile time parameter, either uses the auxiliary apply_cell_flux kernel that does the actual incrementing by gathering the intermediate results from a large temporary array, or uses atomics to do it within the kernel. Both the atomics and the work of the auxiliary kernel was substituted in our code by coloring.

The compute_face_flux kernel is the most computationally intensive among the ones we tested: it uses 165 registers in hierarchical coloring (166 in SoA layout). Also, it achieves, with a block size of 384 and reordered by GPS, $15 \%$ of peak double precision efficiency, compared to the $6-7 \%$ in Airfoil (Table 5). It also uses 8 square root operations and several divides that can't efficiently fill the pipelines at such low occupancy.

The amount of data indirectly accessed by the kernel is also significant: each thread accesses 2 data points indirectly, each holding 32 double precision values. If all of these values are loaded into shared memory, the size exceeds the hardware limits with block sizes larger than 288 ; it didn't run with the original mesh numbering with any block size, and only with smaller block sizes on the reordered meshes (Figure 17). The other measurements were carried out by only loading the incremented data into shared memory. 

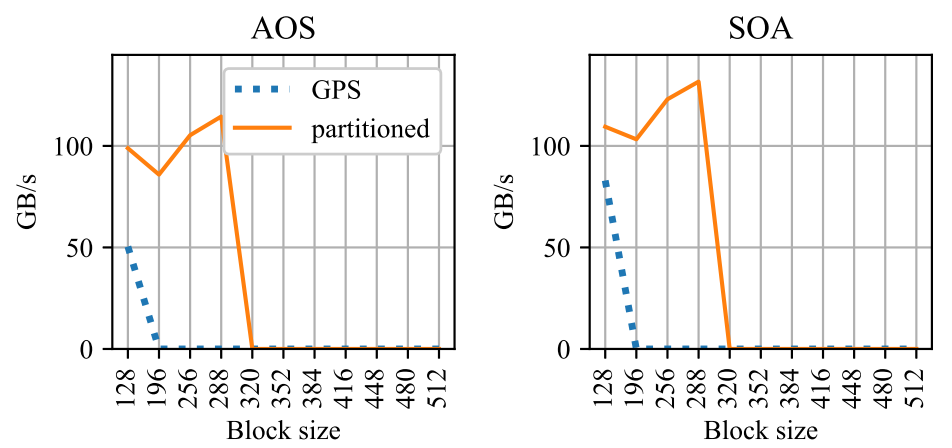

Figure 17: miniAero's compute_face_flux kernel bandwidth on a mesh with 6242304 faces. The kernel didn't run in the cases where the data reuse was not high enough because the large amount of shared memory needed; these are shown here with 0 bandwidth.

The mesh also has a complex structure. 18 and 15 block colors for the GPS reordered and partitioned versions, respectively. The original ordering was far from optimal, we couldn't run the non-reordered version, because the number of block colors exceeded the implementation limit of the library, which is 256 . As with LULESH, only one block was running at a time on each multiprocessor. Although the synchronization overhead was lower (3 and 6 thread colors in the GPS reordered and partitioned versions, respectively), the costly operations prevented high performance gains in the case of the partitioned version (Figure $18)$.

The original Kokkos implementation either used atomic adds or the two-step gathering approach depending on compilation parameters. Our implementation outperformed both with a $75 \%$ speedup (Figure 19). The useful bandwidth in case of the best version of compute_face_flux $(168 \mathrm{~GB} / \mathrm{s})$ reached $34 \%$ of the peak stream bandwidth of the P100 GPU.

On Volta, we achieved $158 \%$ (compared to the atomic version) and $92 \%$ (compared to the two-step version) speedups in the kernel $268 \mathrm{~GB} / \mathrm{s}$ bandwidth (36\% of the peak bandwidth). 

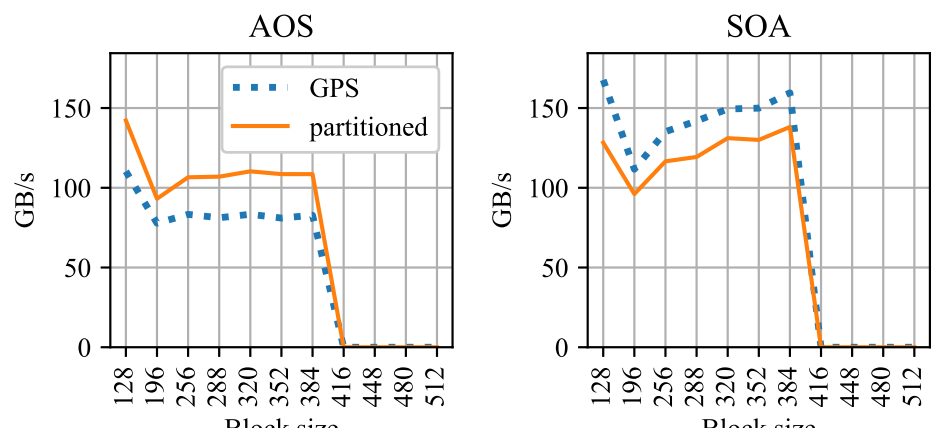

Block size

Block size

Figure 18: miniAero's compute_face_flux kernel bandwidth on a mesh with 6242304 faces. The shared memory was only used to cache the increments, reducing the need for large shared memory size. The kernel didn't fit into the shared memory with block sizes larger than 384 or if not reordered because the large amount of shared memory needed; these are shown here with 0 bandwidth.

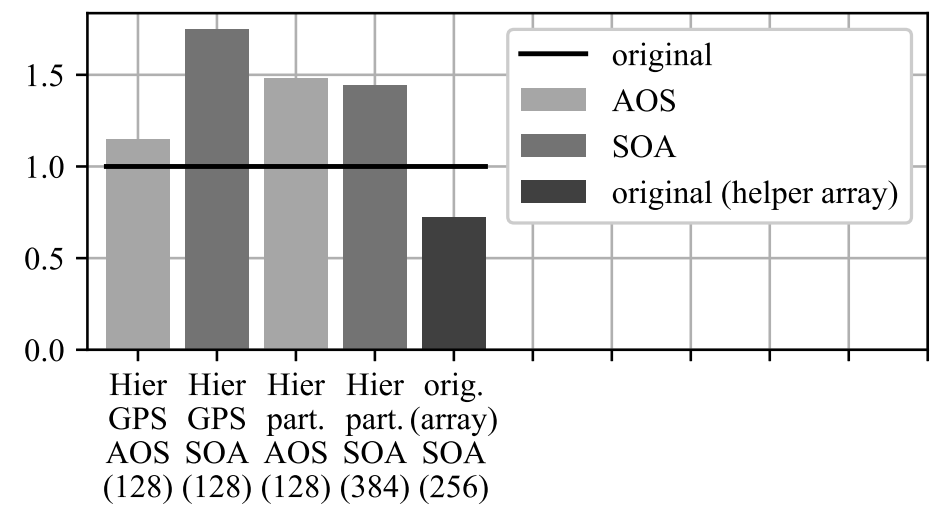

Figure 19: miniAero's compute_face_flux kernel speedup compared to the original code that was using atomic adds, on a mesh with 6242304 faces. The shared memory was only used to cache the increments, reducing the need for large shared memory size. The last bar shows the relative performance of the original code with the helper array approach. 


\begin{tabular}{r|cc|c|c||c|}
\hline Reordering & \multicolumn{2}{|c|}{ GPS } & \multicolumn{2}{c|}{ partition } & original \\
\hline Data layout & AOS & SOA & AOS & SOA & SOA \\
\hline Bandwidth (GB/s) & 67 & 84 & 117 & 113 & 96 \\
Runtime (ms) & 19.09 & 15.38 & 11.04 & 11.38 & 13.39 \\
Achieved Occupancy & 0.06 & 0.06 & 0.12 & 0.12 & 0.23 \\
Global Memory Read Transactions & $73561 \mathrm{k}$ & $82028 \mathrm{k}$ & $53403 \mathrm{k}$ & $77091 \mathrm{k}$ & $87368 \mathrm{k}$ \\
Global Memory Write Transactions & $9153 \mathrm{k}$ & $10390 \mathrm{k}$ & $6694 \mathrm{k}$ & $9008 \mathrm{k}$ & $5934 \mathrm{k}$ \\
Number of Block Colours & 18 & 18 & 15 & 15 & - \\
Number of Thread Colours & 3 & 3 & 6 & 6 & - \\
Reuse Factor & 2.2 & 2.2 & 3.9 & 3.9 & - \\
Issue Stall Reasons (Synchronization) & $4 \%$ & $9 \%$ & $15 \%$ & $21 \%$ & $0 \%$ \\
Issue Stall Reasons (Data Request) & $61 \%$ & $35 \%$ & $47 \%$ & $35 \%$ & $49 \%$ \\
Assue Stall Reasons (Execution Dependency) & $23 \%$ & $33 \%$ & $23 \%$ & $23 \%$ & $13 \%$ \\
Warp Execution Efficiency & $91 \%$ & $84 \%$ & $88 \%$ & $85 \%$ & $91 \%$ \\
FLOP Efficiency(Peak Double) & $5 \%$ & $8 \%$ & $10 \%$ & $12 \%$ & $8 \%$ \\
Block size & \multicolumn{4}{||}{128} & 256 \\
\hline
\end{tabular}

Table 5: Low-level performance metrics of the miniAero's compute_face_flux kernel - hierarchical coloring. The last column details the measured performance of the original code.

\subsubsection{Analysis of structured meshes}

Recalling that the meshes of miniAero and LULESH are actually structured meshes, generated by the code itself allows us to use their structured nature to create partitions with better shapes as opposed to partitions produced by METIS. This in turn allows us to understand the trade-off between high data reuse and number of thread colors. We create 1D (straight line), 2D (rectangles) and 3D (bricks) shape partitions such that these will have an increasing amount of reuse, and with that, number of colors.

While both kernels operate on 3D Cartesian (hex8) meshes, the LULESH kernel IntegrateStressForElems uses a mapping from cells to their connected vertices, and the compute_face_flux kernel in miniAero maps from (internal) faces to cells. We created a number of different partition shapes - 1D lines, 2D rectangles and 3D bricks. Figures 20 and 21 show the bandwidths, reuse factors and the number of thread colors across different block-shapes, along with the result of partitioning the same mesh using METIS. The size of the blocks is 128. The results are from meshes with specifically tailored shapes so that the handcrafted blocks can cover them without any gaps. 
In IntegrateStressForElems, the achieved bandwidth of the original ordering is $134 \mathrm{~GB} / \mathrm{s}$ - it uses a row major order. This is similar to what we use for a line block shape that is 128 cells long and only 1 cell thin in the other dimensions. The partitioned mesh (as detailed in Section 3.1.4) achieved $132 \mathrm{~GB} / \mathrm{s}$, and we achieved $167 \mathrm{~GB} / \mathrm{s}$ bandwidth using our handcrafted blocks, for the same block size (128). Note that using regular shapes is better for the thread coloring algorithm as well. With METIS partitioning, the number of colors needed is higher than in the other cases.

For compute_face_flux, we achieved $167 \mathrm{~GB} / \mathrm{s}$ bandwidth, compared to $101 \mathrm{~GB} / \mathrm{s}$ achieved with METIS partitioning. Of course, using these handcrafted blocks can only be done on meshes that are actually structured, therefore this is not representative of realistic cases. However, these results illustrate clearly that when the number of thread colors are the same, increased reuse leads to better performance. Also, there is an optimal trade off between reuse and the number of thread colors for each application, and performance will suffer above/below that. As an alternative to METIS, we explored using SCOTCH [44] to create the required partitions. However SCOTCH also did not produce a better partitioning for these applications. The challenge lies in finding a partitioning algorithm that can either find the middle ground, or can be tuned along the amount of reuse it aims to achieve. Such partitioning algorithms are not currently available, but the evidence here clearly demonstrate their usefulness.

\section{Conclusion}

In this work we presented a number of novel locality-exploiting optimizations for the efficient execution of unstructured-mesh algorithms on GPUs. The key focus was to improve performance of kernels with indirect increment data-access patterns. We build on well known techniques such as data-layouts (AoS and 


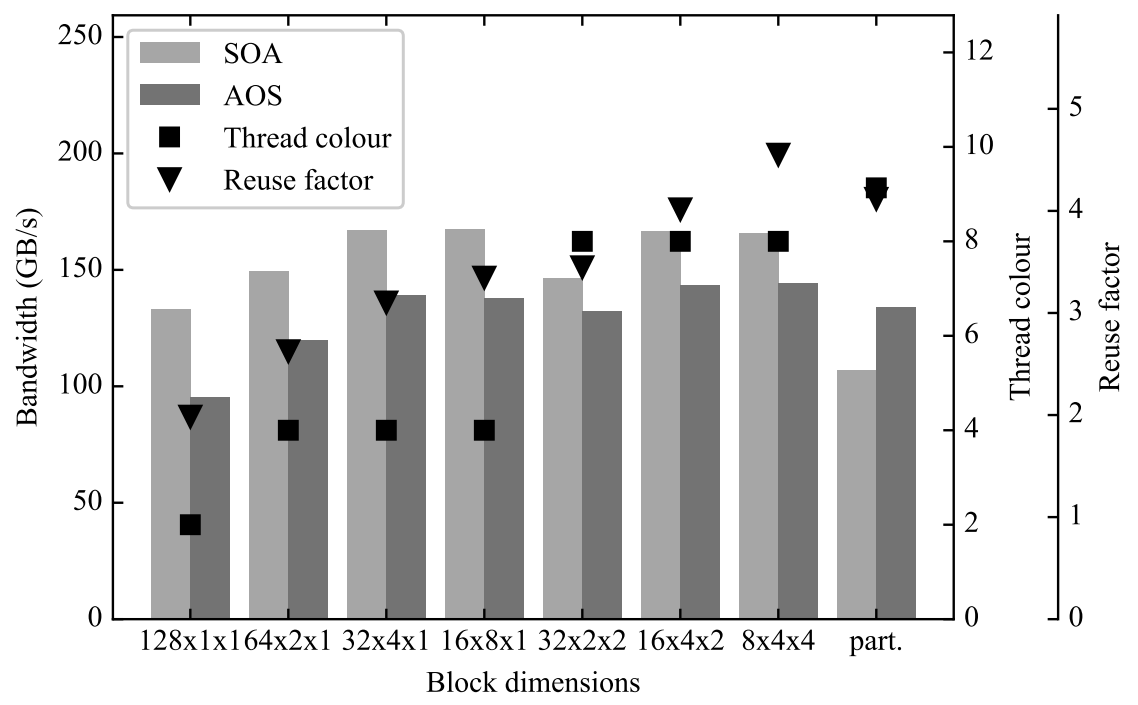

Figure 20: LULESH's IntegrateStressForElems kernel with explicitly controlled partitioning. For comparison, the last column shows the result on the same mesh, partitioned by METIS.

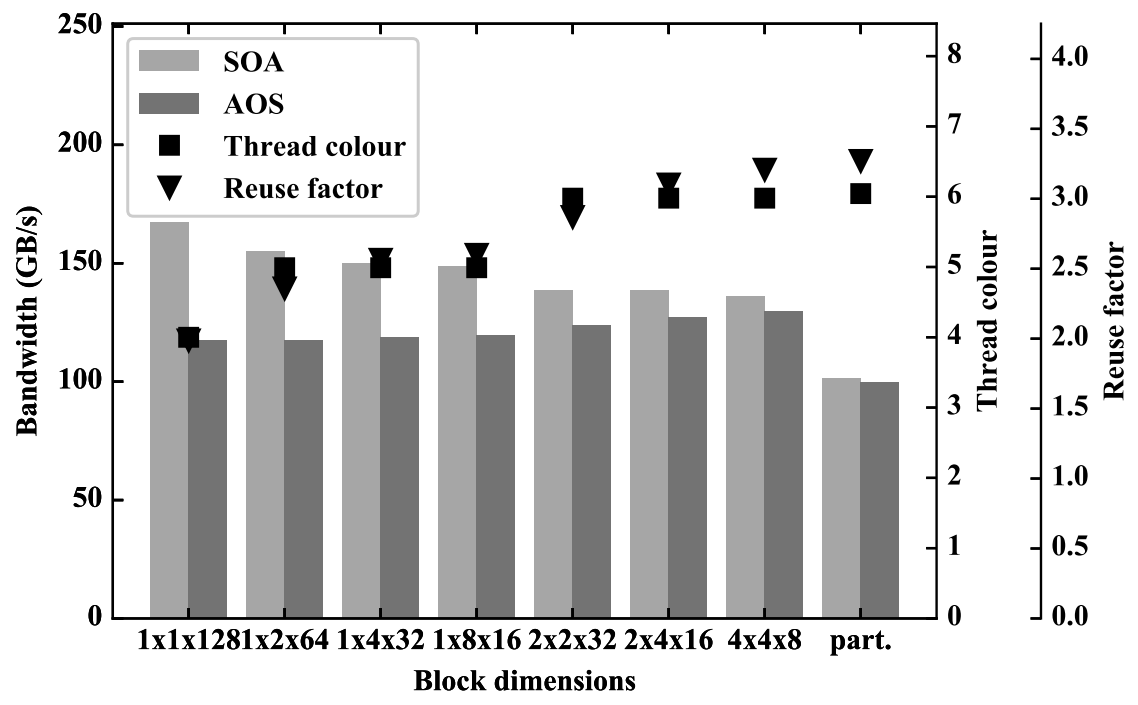

Figure 21: miniAero's compute_face_flux kernel with explicitly controlled partitioning. For comparison, the last column shows the result on the same mesh, partitioned by METIS. 
SoA), graph bandwidth minimizing algorithms, global and hierarchical coloring approaches for exploring efficient execution strategies. A novel reordering algorithm which uses k-way recursive partitioning, together with the use of GPU shared memory implementing a hierarchical coloring method is designed to significantly improve data reuse within CUDA thread blocks.

The new optimizations are then applied to several well established unstructured mesh applications, investigating their performance on NVIDIA's latest P100 and V100 GPUs. A range of performance metrics were benchmarked including runtime, memory transactions, achieved bandwidth performance, GPU occupancy and data reuse factors and are used to understand and explain the key factors impacting performance.

When comparing the performance of global coloring to that of hierarchical coloring (with shared memory), we demonstrated that the latter approach consistently performed better. This was due to its ability to exploit the temporal locality in indirectly accessed data by avoiding data races in shared memory with synchronization within thread blocks rather than different kernel launches.

Analyzing the performance of reordering based on GPS renumbering and partitioning showed that former improves global coloring with increasing spatial reuse, while the latter can significantly improve the shared memory approach by increasing data reuse within thread blocks. In this case, we see smaller shared memory usage and fewer global memory transactions.

We also see that there is a trade-off between high data reuse and large numbers of thread colors in hierarchical coloring. This is especially pronounced in 3D applications, and when the achieved occupancy is low: the more thread colors a block has, the more synchronizations it will need, the latency of which can be hard to hide when there are few eligible warps.

The locality exploiting optimizations detailed in this paper enable us to im- 
prove performance of indirect kernels by $19 \%$ on Airfoil, $20 \%$ on Volna, $15 \%$ on Bookleaf, $75 \%$ on Lulesh and $75 \%$ on miniAero over the original implementations, on the GPUs tested. These results advances the state of the art, demonstrating that the algorithmic patterns used in most current implementations (particularly in case of US DoE codes represented by LULESH and MiniAero) could be significantly improved upon by the adoption of two-level coloring schemes and partitioning for increased data reuse.

When carrying out this work, it had become clear that partitioning algorithms in traditional libraries such as METIS and SCOTCH were not particularly well suited for producing such small partition sizes. As potential future work, we wish to explore algorithms that are better optimized for this purpose. The performance of these partitioning algorithms was also low - parallelizing this could be another interesting challenge. Finally, we are planning to integrate these algorithms into the OP2 library, so they can be automatically deployed on applications that already use the OP2 library, such as Airfoil, BookLeaf, Volna or Rolls-Royce Hydra.

The optimized algorithms are implemented as an open-source software library [3].

\section{Acknowledgements}

István Reguly was supported by the János Bolyai Research Scholarship of the Hungarian Academy of Sciences. Project no. PD 124905 has been implemented with the support provided from the National Research, Development and Innovation Fund of Hungary, financed under the PD_17 funding scheme. Additionally, this research was supported in part by the UK Engineering and Physical Sciences Research Council (EPSRC) grant EP/S005072/1 - Strategic Partnership in Computational Science for Advanced Simulation (ASiMoV). Gi- 
han Mudalige was supported by the Royal Society Industrial Fellowship Scheme (INF/R1/180012). The authors would like to acknowledge the use of the University of Oxford Advanced Research Computing (ARC) facility in carrying out this work http://dx.doi.org/10.5281/zenodo.22558. The research has been supported by the European Union, co-financed by the European Social Fund (EFOP-3.6.2-16-2017-00013).

[1] Hydrodynamics Challenge Problem, Lawrence Livermore National Laboratory, Tech. Rep. LLNL-TR-490254.

[2] miniAero CFD Mini-Application, https://github.com/Mantevo/ miniAero.

[3] Software repository of our implementation, https://github.com/bgd54/ cuda_fot.

[4] P. Colella, Defining software requirements for scientific computing, DARPA HPCS presentation, 2004.

[5] OpenCFD, OpenFOAM - The Open Source CFD Toolbox - User's Guide, OpenCFD Ltd., United Kingdom, 1st Edition (11 Apr. 2007).

[6] P. Moinier, J. Muller, M. B. Giles, Edge-based multigrid and preconditioning for hybrid grids, AIAA journal 40 (10) (2002) 1954-1960.

[7] R. T. Biedron, J.-R. Carlson, J. M. Derlaga, P. A. Gnoffo, D. P. Hammond, W. T. Jones, B. Kleb, E. M. Lee-Rausch, E. J. Nielsen, M. A. Park, et al., Fun3d manual: 13.1.

[8] D. J. Mavriplis, Parallel performance investigations of an unstructured mesh navier-stokes solver, The International Journal of High Performance Computing Applications 16 (4) (2002) 395-407. 
[9] H.-Q. Jin, M. Frumkin, J. Yan, The openmp implementation of nas parallel benchmarks and its performance.

[10] Z. Nagy, C. Nemes, A. Hiba, Á. Csík, A. Kiss, M. Ruszinkó, P. Szolgay, Accelerating unstructured finite volume computations on field-programmable gate arrays, Concurrency and Computation: Practice and Experience 26 (3) (2014) 615-643.

[11] T. Akamine, K. Inakagata, Y. Osana, N. Fujita, H. Amano, Reconfigurable out-of-order mechanism generator for unstructured grid computation in computational fluid dynamics, in: Field Programmable Logic and Applications (FPL), 2012 22nd International Conference on, IEEE, 2012, pp. 136-142.

[12] M. Heroux, R. Bartlett, V. H. R. Hoekstra, J. Hu, T. Kolda, R. Lehoucq, K. Long, R. Pawlowski, E. Phipps, A. Salinger, H. Thornquist, R. Tuminaro, J. Willenbring, A. Williams, An Overview of Trilinos, Tech. Rep. SAND2003-2927, Sandia National Laboratories (2003).

[13] S. Balay, S. Abhyankar, M. F. Adams, J. Brown, P. Brune, K. Buschelman, L. Dalcin, A. Dener, V. Eijkhout, W. D. Gropp, D. Kaushik, M. G. Knepley, D. A. May, L. C. McInnes, R. T. Mills, T. Munson, K. Rupp, P. Sanan, B. F. Smith, S. Zampini, H. Zhang, H. Zhang, PETSc Web page, http: //www.mcs.anl.gov/petsc (2018).

URL http://www.mcs.anl.gov/petsc

[14] Z. DeVito, N. Joubert, F. Palacios, S. Oakley, M. Medina, M. Barrientos, E. Elsen, F. Ham, A. Aiken, K. Duraisamy, et al., Liszt: a domain specific language for building portable mesh-based pde solvers, in: Proceedings of 2011 International Conference for High Performance Computing, Networking, Storage and Analysis, ACM, 2011, p. 9. 
[15] M. Giles, G. Mudalige, I. Reguly, Op2 airfoil example.

[16] P. Vincent, F. Witherden, B. Vermeire, J. S. Park, A. Iyer, Towards green aviation with python at petascale, in: SC16: International Conference for High Performance Computing, Networking, Storage and Analysis, 2016, pp. 1-11. doi:10.1109/SC.2016.1.

[17] I. Z. Reguly, M. B. Giles, Finite element algorithms and data structures on graphical processing units, International Journal of Parallel Programming 43 (2) (2015) 203-239. doi:10.1007/s10766-013-0301-6.

URL https://doi.org/10.1007/s10766-013-0301-6

[18] E. Elsen, P. LeGresley, E. Darve, Large calculation of the flow over a hypersonic vehicle using a gpu, Journal of Computational Physics 227 (24) (2008) 10148 - 10161. doi:https://doi.org/10.1016/j.jcp.2008.08.023.

URL http://www.sciencedirect.com/science/article/pii/ S0021999108004476

[19] J. Cohen, M. J. Molemaker, A fast double precision cfd code using cuda, Parallel Computational Fluid Dynamics: Recent Advances and Future Directions (2009) 414-429.

[20] J.-F. Remacle, R. Gandham, T. Warburton, Gpu accelerated spectral finite elements on all-hex meshes, Journal of Computational Physics 324 (2016) 246-257.

[21] M. J. Castro, S. Ortega, M. De la Asuncion, J. M. Mantas, J. M. Gallardo, Gpu computing for shallow water flow simulation based on finite volume schemes, Comptes Rendus Mécanique 339 (2-3) (2011) 165-184.

[22] B. Wu, Z. Zhao, E. Z. Zhang, Y. Jiang, X. Shen, Complexity analysis and 
algorithm design for reorganizing data to minimize non-coalesced memory accesses on gpu, in: ACM SIGPLAN Notices, ACM, 2013, pp. 57-68.

[23] Z. Fu, T. J. Lewis, R. M. Kirby, R. T. Whitaker, Architecting the finite element method pipeline for the gpu, Journal of computational and applied mathematics 257 (2014) 195-211.

[24] I. Karlin, J. Keasler, R. Neely, Lulesh 2.0 updates and changes, Tech. Rep. LLNL-TR-641973 (August 2013).

[25] D. Truby, S. Wright, R. Kevis, S. Maheswaran, A. Herdman, S. Jarvis, Bookleaf: An unstructured hydrodynamics mini-application, in: 2018 IEEE International Conference on Cluster Computing (CLUSTER), 2018, pp. 615-622. doi:10.1109/CLUSTER.2018.00078.

[26] A. B. Williams, Cuda/gpu version of minife mini-application., Tech. rep., Sandia National Lab.(SNL-NM), Albuquerque, NM (United States) (2012).

[27] C. R. Ferenbaugh, Pennant: an unstructured mesh mini-app for advanced architecture research, Concurrency and Computation: Practice and Experience 27 (17) (2015) 4555-4572. arXiv:https://onlinelibrary.wiley.com/doi/pdf/10.1002/cpe.3422, doi:10.1002/cpe.3422.

URL https://onlinelibrary.wiley.com/doi/abs/10.1002/cpe.3422

[28] G. Mudalige, M. Giles, I. Reguly, C. Bertolli, P. Kelly, Op2: An active library framework for solving unstructured mesh-based applications on multi-core and many-core architectures, in: Innovative Parallel Computing (InPar), 2012, IEEE, 2012, pp. 1-12.

[29] T. Zegard, G. H. Paulino, Toward gpu accelerated topology optimization on unstructured meshes, Structural and Multidisciplinary Optimization 48 (3) 
(2013) 473-485. doi:10.1007/s00158-013-0920-y.

URL https : //doi.org/10.1007/s00158-013-0920-y

[30] J. Kraus, M. Schlottke, A. Adinetz, D. Pleiter, Accelerating a c ++ cfd code with openacc, in: Proceedings of the First Workshop on Accelerator Programming Using Directives, WACCPD '14, IEEE Press, Piscataway, NJ, USA, 2014, pp. 47-54. doi:10.1109/WACCPD.2014.11.

URL http://dx.doi .org/10.1109/WACCPD. 2014.11

[31] K. Sharma, I. Karlin, J. Keasler, J. R. McGraw, V. Sarkar, Data layout optimization for portable performance, in: European Conference on Parallel Processing, Springer, 2015, pp. 250-262.

[32] J. Norman E. Gibbs, William G. Poole, P. K. Stockmeyer, An algorithm for reducing the bandwidth and profile of a sparse matrix, SIAM Journal on Numerical Analysis 13. doi:10.2307/2156090.

[33] G. Karypis, V. Kumar, A fast and high quality multilevel scheme for partitioning irregular graphs, SIAM Journal on scientific Computing 20 (1) (1998) 359-392.

[34] G. Karypis, K. Schloegel, V. Kumar, Parmetis: Parallel graph partitioning and sparse matrix ordering library, Version 1.0, Dept. of Computer Science, University of Minnesota.

[35] D. LaSalle, G. Karypis, Efficient nested dissection for multicore architectures, in: European Conference on Parallel Processing, Springer, 2015, pp. $467-478$.

[36] C. NVIDIA, Nvidia tesla p100 (2016).

URL https://images.nvidia.com/content/pdf/tesla/whitepaper/

pascal-architecture-whitepaper.pdf 
[37] C. NVIDIA, Nvidia tesla v100 gpu architecture (2017).

URL https://images.nvidia.com/content/volta-architecture/pdf/ volta-architecture-whitepaper.pdf

[38] OP2 github repository, https://github.com/OP2/OP2-Common.

[39] D. Dutykh, R. Poncet, F. Dias, The volna code for the numerical modeling of tsunami waves: Generation, propagation and inundation, European Journal of Mechanics-B/Fluids 30 (6) (2011) 598-615.

[40] I. Z. Reguly, D. Giles, D. Gopinathan, L. Quivy, J. H. Beck, M. B. Giles, S. Guillas, , F. Dias, The volna-op2 tsunami code (version 1.5), Geosci. Model Dev., 11. doi:10.5194/gmd-11-4621-2018.

[41] Uk mini-app consortium, https://uk-mac.github.io.

[42] M. A. Heroux, D. W. Doerfler, P. S. Crozier, J. M. Willenbring, H. C. Edwards, A. Williams, M. Rajan, E. R. Keiter, H. K. Thornquist, R. W. Numrich, Improving Performance via Mini-applications, Tech. Rep. SAND20095574, Sandia National Laboratories (2009).

[43] H. C. Edwards, C. R. Trott, D. Sunderland, Kokkos: Enabling manycore performance portability through polymorphic memory access patterns, Journal of Parallel and Distributed Computing 74 (12) (2014) 3202 3216, domain-Specific Languages and High-Level Frameworks for HighPerformance Computing. doi:https://doi.org/10.1016/j.jpdc.2014.07.003.

URL http://www.sciencedirect.com/science/article/pii/ S0743731514001257

[44] F. Pellegrini, J. Roman, Scotch: A software package for static mapping by dual recursive bipartitioning of process and architecture graphs, 
in: Proceedings of the International Conference and Exhibition on HighPerformance Computing and Networking, HPCN Europe 1996, SpringerVerlag, Berlin, Heidelberg, 1996, pp. 493-498. 